1

\title{
Dynamic analyses of operating offshore wind turbines including
}

\section{soil-structure interaction}

\author{
Haoran Zuo, Kaiming Bi ${ }^{*}, 1$, Hong Hao*,2
}

Centre for Infrastructure Monitoring and Protection, School of Civil and Mechanical Engineering, Curtin University, Kent Street, Bentley, WA 6102, Australia

${ }^{*, 1}$ Corresponding author; ${ }^{*, 2}$ Principal corresponding author.

E-mail address: haoran.zuo@postgrad.curtin.edu.au (H.Zuo); kaiming.bi@curtin.edu.au (K. Bi);

hong.hao@curtin.edu.au (H.Hao).

\section{ABSTRACT}

In the dynamic analyses of offshore wind turbines subjected to the external vibration sources, the wind turbines are normally assumed in the parked condition and the blades are considered by a lumped mass located at the top of the tower. In reality, the geometrical characteristics and rotational velocity of the blades can directly influence the wind loads acting on the blades. Moreover, the centrifugal stiffness generated by the rotating blades can increase the stiffness and natural frequencies of the blades, which in turn can further affect the structural responses. The lumped mass model, therefore, may lead to inaccurate structural response estimations. On the other hand, monopile, a long hollow steel member inserting into the water and sea bed, is generally designed as the foundation of an offshore wind turbine. The soil-monopile interaction can further alter the vibration characteristics and dynamic responses of offshore wind turbines. In the present study, the dynamic responses of the modern NREL 5 MW wind turbine subjected to the combined wind and sea wave loadings are numerically investigated by using the finite element code ABAQUS. The blades are explicitly modelled and soil-structure interaction (SSI) is considered. The influences of operational condition and rotor velocity on the dynamic behaviours are systematically investigated. It is found that the responses of the wind turbine in the operating condition are much larger than those in the parked 

plane vibrations of the blades.

Keywords: Offshore wind turbine, operational condition, SSI, rotor velocity

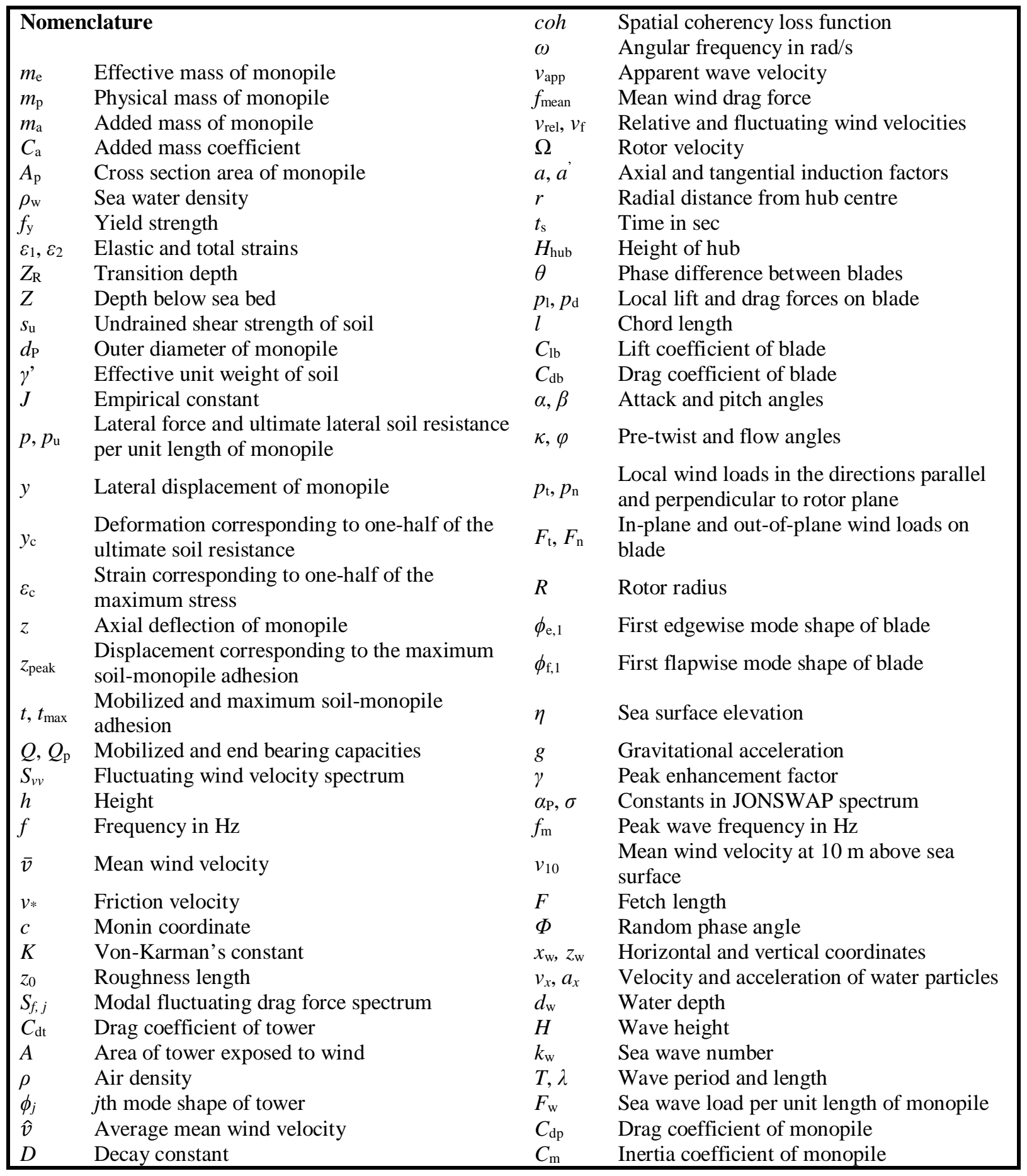




\section{Introduction}

34 Offshore wind turbines play an important role in producing electrical energy. Multi-megawatt offshore wind turbines with slender tower and large rotor are widely adopted in the state-of-the-art designs to more efficiently extract the vast wind energy resources. For example, the tower height and rotor radius of the modern NREL $5 \mathrm{MW}$ horizontal axis wind turbine reach $87.6 \mathrm{~m}$ and $63 \mathrm{~m}$ respectively [1]. These flexible wind turbines are vulnerable to the external vibration sources. For example, wind and sea wave loadings, which are experienced constantly during the whole lifetime of an offshore wind turbine, can result in excessive vibrations to the structures. These adverse vibrations may compromise the wind energy output, cause the fatigue damage to the structural components, and even direct structural damage under extreme conditions. To ensure the safe and effective operations of these offshore wind turbines, it is important to accurately understand the dynamic behaviours when they are subjected to the external vibration loadings.

Extensive research works have been conducted by different researchers to investigate the dynamic behaviours of wind turbines under wind, sea wave and/or seismic loadings. To simplify the analysis, the wind turbines were normally assumed in the parked condition, and the blades were modelled as a lumped mass located at the top of the tower [2-8] by neglecting the geometrical configurations of the blades and the interaction between the tower and blades. In reality, the geometrical characteristics and rotational velocity of the blades can directly influence the wind loads acting on the blades [9]. Moreover, the geometry of the rotor can influence the vibration characteristics of the wind turbine especially when it is in the operating condition since the locations of the blades are changing periodically and the centrifugal stiffness generated by the rotating blades can increase the stiffness and the natural frequencies of the blades [10], which in turn can indirectly affect the dynamic responses of wind turbines. The simplified lumped mass model therefore may lead to the inaccurate structural response estimations. To investigate the influence of blades on the dynamic behaviours of wind turbines, Prowell et al. [11], Kjørlaug and Kaynia [12] and Santangelo et al. [13] considered the geometrical characteristics of the blades and explicitly developed the finite element (FE) models of the blades in the seismic analyses of wind turbines. However, only the parked condition was considered in these studies, rotating induced 
blades location changes and stiffness increment therefore were not considered. To investigate the dynamic behaviours of operating wind turbines, Prowell et al. [14] performed shaking table tests to investigate its seismic responses, additional damping in the fore-aft direction was observed compared to the parked condition. Some researchers simplified each blade as a single [15, 16] or two [17] degrees-of-freedom (DOF) system, and the structural responses were estimated by using the homemade programs (e.g. in MATLAB). A lot of mathematics are involved in the calculations, these methods are therefore not convenient for other researchers/engineers to use. Moreover, wind loads acting along the height of the tower and the length of the blades are inevitably different, hence the structural responses may not be realistically captured by these simplified models. Some other researchers modelled the wind turbines by using the commercially available software such as FAST (e.g. [18]) or validated their models against FAST [19]. The structural components can be explicitly developed and the blades rotation can be considered by using FAST. However, as indicated in the user's guide [20], FAST employs a combination of modal and multi-body dynamics formulations and models the blades and tower as flexible elements using a linear modal representation that assumes small deflections. In other words, FAST can only simulate the elastic response of wind turbines. Under the extreme loading conditions, the wind turbine may experience nonlinear deformations, which may not be realistically considered by FAST.

On the other hand, the monopile is widely designed as the foundation of offshore wind turbines due to its simplicity [21, 22]. A typical monopile is a long hollow steel member with 3-6 m outer diameter and 22-40 m length [6], inserting into the sea water and sea bed. It can be regarded as an extension of the wind turbine tower. For such a slender flexible foundation, the interaction between the monopile and the surrounding soil is inevitable and can reduce the vibration frequencies or even vibration modes of the structure, which in turn may further influence the dynamic behaviours of offshore structures [23]. Many numerical [24, 25] and experimental [26, 27] studies have been carried out to investigate the influence of SSI on the vibration characteristics of wind turbines. Andersen et al. [24] and Arany et al. [25] investigated the effect of soil uncertainty on the first natural frequency of offshore wind turbine; Lombardi et al. [26] and Bhattacharya and Adhikari [27] conducted laboratory tests on a scaled wind turbine model and found that the natural frequencies of wind turbine were 
strongly related to the foundation flexibility. Some researchers also investigated the influence of soilstructure interaction (SSI) on the dynamic responses of wind turbines [6, 7, 12, 13, 16, 17, 21, 28]. However, it should be noted that in all these studies the wind turbines were either assumed in the parked condition $[12,13]$ and the blades were lumped at the tower top $[6,7,28]$, or the rotation of the blades was considered by the simplified 1 - or 2 -DOF systems $[16,17,21]$. The influence of blades on the structural responses was therefore not realistically considered as discussed above.

In the present study, a detailed FE model of the modern NREL 5 MW wind turbine is developed by using the commercially available finite element code ABAQUS. The tower and blades are explicitly modelled. Compared to the previous simplified models, the present numerical model can realistically consider the influence of geometrical configurations of the blades on the wind loads, as well as the centrifugal stiffness variations of the blades generated by the blades rotation. Moreover, the possible nonlinear behaviour of the tower and blades can also be conveniently considered. This FE model can be readily used by other researchers/engineers. This detailed FE model is used to systematically investigate the influences of operational conditions and SSI on the wind turbine responses when subjected to the combined actions of wind and sea wave loadings. The structure of this paper is organized as follows: the NREL 5 MW wind turbine and the development of the FE model is presented in Section 2; Section 3 defines the vibration sources including the wind and sea wave loadings which are used in the analyses; the numerical results are discussed in Section 4 and some concluding remarks are made in Section 5.

\section{Numerical model}

\subsection{NREL 5 MW wind turbine}

The modern NREL 5 MW three-bladed wind turbine is selected as an example in the present study. The wind turbine is selected simply because its properties are well defined in many previous studies such as in [1]. The outer diameters at the top and bottom of the tower are $3.87 \mathrm{~m}$ and $6 \mathrm{~m}$, and the corresponding wall thickness are $0.019 \mathrm{~m}$ and $0.027 \mathrm{~m}$ respectively. The outer diameter and wall thickness decrease linearly from the bottom to the tower top. The total length of the monopile is $75 \mathrm{~m}$, in which $20 \mathrm{~m}$ and $45 \mathrm{~m}$ are in the water and sea bed respectively and another $10 \mathrm{~m}$ is above the mean 
sea level [29]. The diameter and wall thickness of the monopile foundation are the same as the bottom cross section of the tower. The radius of the hub is $1.5 \mathrm{~m}$ and the length of the blade is $61.5 \mathrm{~m}$. The distance from the hub centre to the tip of the blade is therefore $63 \mathrm{~m}$.

The pre-twisted blade is made up of eight unique airfoil sections and the geometries can be found in [1]. The mass of each blade is $17,740 \mathrm{~kg}$ as reported [1], but the wall thickness of the blade is not given in [1]. A uniform wall thickness is assumed in the present study and a thickness of $0.019 \mathrm{~m}$ is computed to ensure that the mass of the blade is the same as that reported in [1]. Fig. 1 shows the main dimensions of the wind turbine and Table 1 tabulates the detailed information.

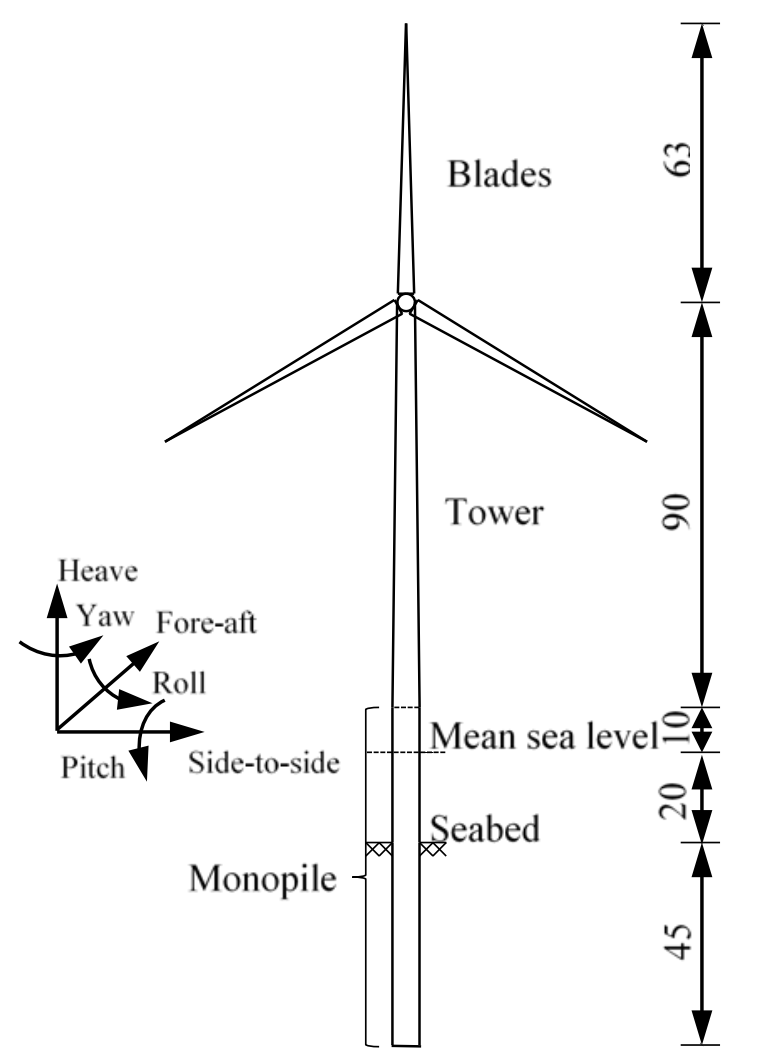

Fig. 1. Offshore wind turbine model (Front view, dimensions in $\mathrm{m}$ )

\subsection{Finite element model}

The detailed three-dimensional (3D) FE model of the NREL 5 MW wind turbine is developed by using the finite element code ABAQUS. The tower and monopile above and in the sea water are modelled by the shell elements (S4 in ABAQUS), while the monopile buried in the soil medium is 
Hub and Nacelle

Tower released.

\section{Table 1}

modelled by the beam elements (B31 in ABAQUS). The nacelle and hub are fixed at the top of the tower, only the masses of them are considered in the numerical model, and they are modelled by the point mass element in ABAQUS and is lumped at the tower top. To ensure the deformation continuity at the connection between the tower and the monopile, the cross sections of the bottom of the tower and the top of the monopile are tied with each other. To consider the influence of blades on the dynamic behaviours of offshore wind turbines, the blades are explicitly developed and they are modelled by the shell elements again. A hinge connection between the tower and blades is defined to simulate the rotation of the blades and the rotational DOF along the out-of-rotor-plane direction is

Properties of NREL 5MW wind turbine [1]

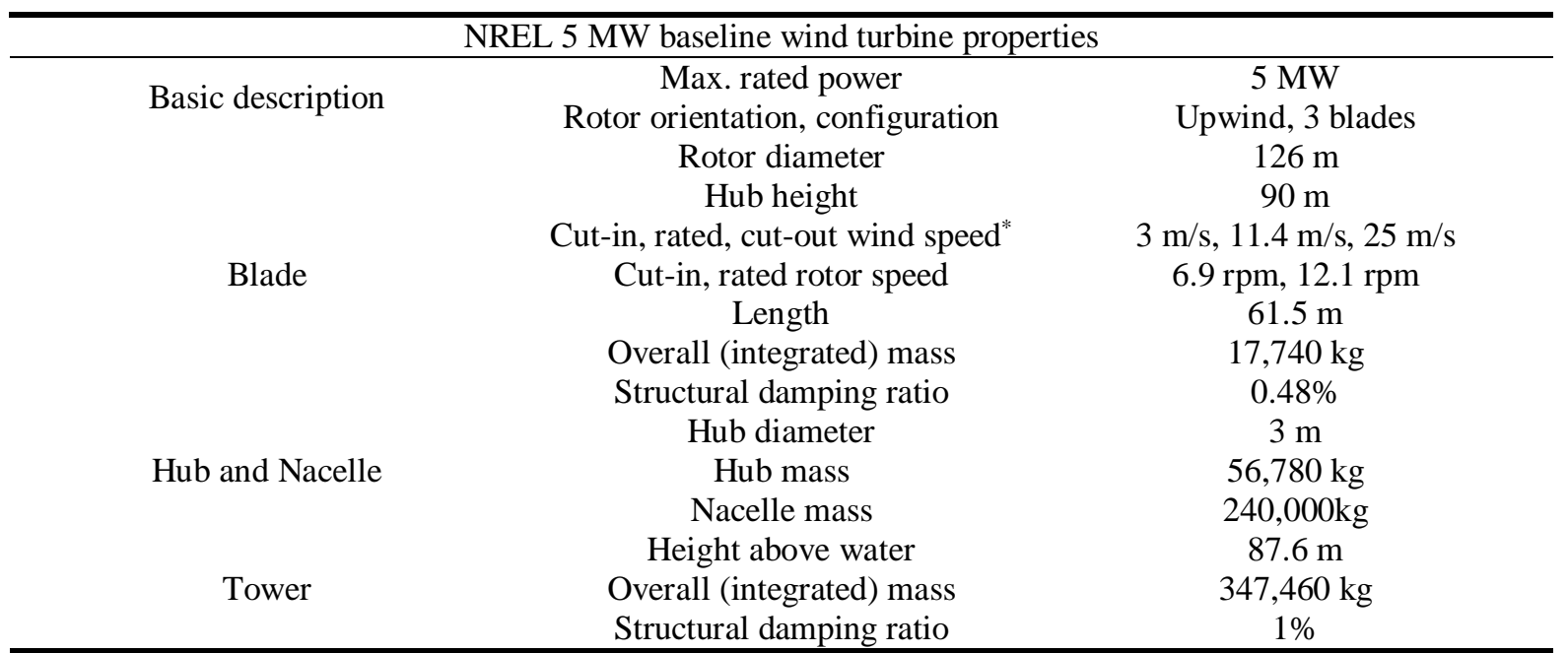

*In Table 1, cut-in wind speed means that wind turbine starts to rotate at a (cut-in) rotor speed of 6.9 rpm; rated wind speed means the maximum energy output of wind turbine will be achieved at a (rated) rotor speed of 12.1 rpm and cut-out wind speed is the speed above which the wind turbine stops working in order to protect the electrical and mechanical components.

The cross sections of the blades, tower and monopile in the water are divided into 24 elements as suggested in [30]. A convergence test shows that an element size of $1 \mathrm{~m}$ along the blades, tower and monopile in the water and soil yields a good balance between the computational time and accuracy, an element size of $1 \mathrm{~m}$ is therefore selected in these directions. As mentioned above, the monopile above and in the sea water is modelled by the shell elements while the monopile in the soil is modelled by 
the beam elements in order to conveniently consider SSI. To make sure the same deformations of the beam element and shell elements at the sea bed level, the node of the beam element and nodes of the shell elements are coupled with each other at this cross section. Fig. 2 shows FE model of the wind turbine except the monopile in the soil medium, the modelling of which will be discussed in Section 2.3. In the numerical model, the three blades are labelled as \#1 to \#3 in an anticlockwise direction as shown in Fig. 2.

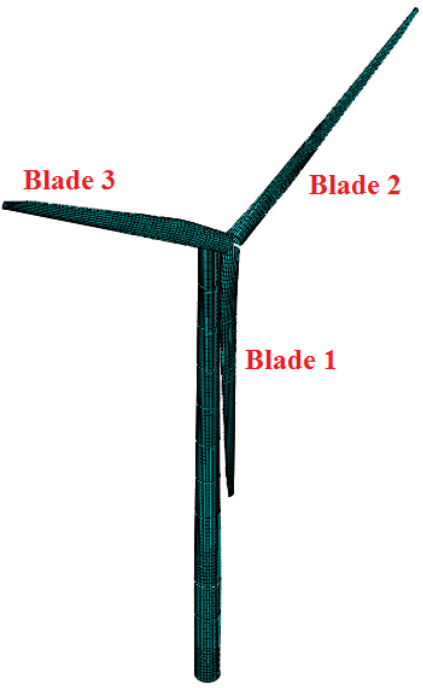

(a) Wind turbine in the parked condition

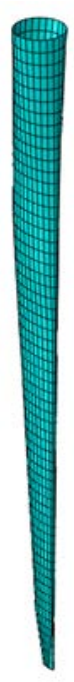

(b) Blade

Fig. 2. FE model of the wind turbine (the monopile in the soil medium is not shown)

The blades are made of polyester with a density of $1850 \mathrm{~kg} / \mathrm{m}^{3}$ [31]. The tower and monopile are made of steel. For the monopile above the mean sea level and buried in the soil medium, the density is $7850 \mathrm{~kg} / \mathrm{m}^{3}$, while the density of the tower is taken as $8500 \mathrm{~kg} / \mathrm{m}^{3}$ [1] to account for the paint, welds, bolts and flanges that are not directly considered in the numerical model. For the monopile in the sea water, the vibrating monopile can impart an acceleration to the surrounding sea water. The watermonopile interaction is modelled by the added mass method (e.g. [30]) in the present study, in which the effective mass $m_{\mathrm{e}}$ of the monopile can be expressed as

$$
m_{e}=m_{p}+m_{a}
$$


171 where $m_{\mathrm{p}}$ is the monopile physical mass and $m_{\mathrm{a}}$ denotes the added mass which can be calculated as $172[32]$

$$
m_{a}=C_{a} A_{p} \rho_{w}
$$

173 where $A_{\mathrm{p}}$ is the cross section area of the monopile; $\rho_{\mathrm{w}}=1030 \mathrm{~kg} / \mathrm{m}^{3}$ is the sea water density and $C_{\mathrm{a}}$ is 174 the added mass coefficient, which is assumed as 1.0 in the present study [32]. The effective density of

175 the monopile in the sea water is therefore $8880 \mathrm{~kg} / \mathrm{m}^{3}$. Table 2 tabulates the material properties of the 176 blades, tower and monopile. The polyester and steel are assumed as ideal elastic-plastic materials and 177 the relationship between stress and strain is shown in Fig. 3. As shown in Fig. 3, $f_{\mathrm{y}}$ is the yield strength, $\varepsilon_{1}$ is the elastic strain and $\varepsilon_{2}$ is the strain which equals to the sum of the elastic and plastic strains.

Table 2

Material properties of the wind turbine $[1,31]$

\begin{tabular}{|c|c|c|c|c|c|c|}
\hline Component & Material & $\begin{array}{l}\text { Density } \\
\left(\mathrm{kg} / \mathrm{m}^{3}\right)\end{array}$ & $\begin{array}{c}\text { Young's } \\
\text { modulus (GPa) }\end{array}$ & $\begin{array}{l}\text { Poisson’s } \\
\text { ratio }\end{array}$ & $\begin{array}{l}\text { Yield strength } \\
\text { (MPa) }\end{array}$ & $\begin{array}{l}\text { Plastic } \\
\text { strain }\end{array}$ \\
\hline Blade & Polyester & 1850 & 38 & 0.3 & 700 & 0.02 \\
\hline Tower & Steel & 8500 & 210 & 0.3 & 235 & 0.01 \\
\hline Monopile in the water & Steel & 8880 & 210 & 0.3 & 235 & 0.01 \\
\hline $\begin{array}{l}\text { Monopile above water } \\
\text { and in the soil }\end{array}$ & Steel & 7850 & 210 & 0.3 & 235 & 0.01 \\
\hline
\end{tabular}

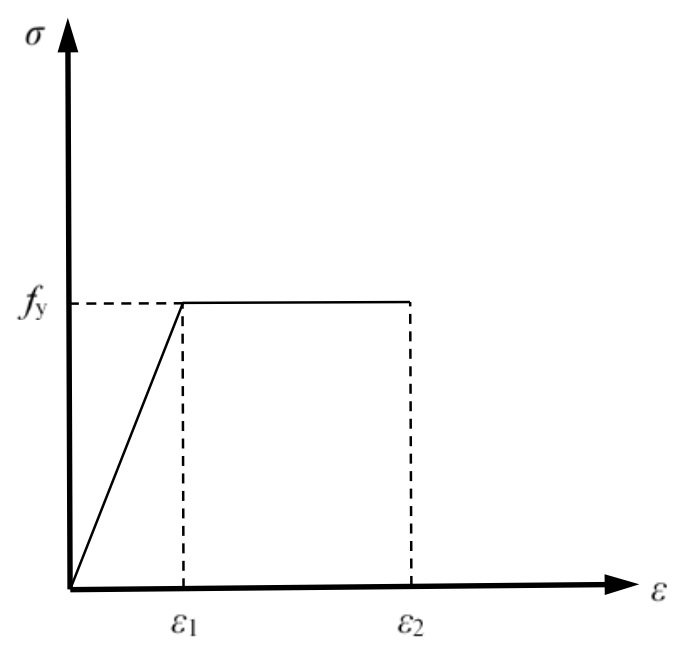


Some previous studies (e.g. [1, 19]) calculated the vibration frequencies and vibration modes of the NREL 5 MW wind turbine in the parked condition and without considering SSI. To examine the accuracy of the developed FE model, the natural frequencies and vibration modes of the wind turbine are calculated and compared with those in a previous study [1]. For a fair comparison, the parked condition is considered and the bottom of the tower is fixed (i.e. SSI is not considered) in this section. Table 3 tabulates the first 12 natural frequencies, the corresponding vibration modes and the differences of the vibration frequencies between these two studies. It can be seen that in general good agreements are observed. Slightly larger differences are obtained when the frequencies are corresponding to the vibration modes of the blades. This is because the strengthening webs in the blades are not included in the numerical model due to the lack of the detailed geometry and material properties. The first flapwise and edgewise vibration mode shapes of the blade are compared with those in another study [33] and the results are shown in Fig. 12 in Section 3.2. Good agreements are obtained again and the slightly larger amplitudes in the present model are because the strengthening webs are not modelled as discussed above.

Table 3

Natural frequencies of NREL 5 MW wind turbine in the parked condition and without considering SSI

\begin{tabular}{ccccc}
\hline Mode & Description & {$[1](\mathrm{Hz})$} & Current study $(\mathrm{Hz})$ & Difference \\
\hline 1 & $1^{\text {st }}$ tower side-to-side & 0.312 & 0.300 & $-3.85 \%$ \\
2 & $1^{\text {st }}$ tower fore-aft & 0.324 & 0.316 & $-2.47 \%$ \\
3 & $1^{\text {st }}$ blade flapwise yaw & 0.666 & 0.490 & $-26.43 \%$ \\
4 & $1^{\text {st }}$ blade flapwise pitch & 0.668 & 0.541 & $-19.01 \%$ \\
5 & $1^{\text {st }}$ blade collective flap & 0.699 & 0.607 & $-13.16 \%$ \\
6 & $1^{\text {st }}$ blade edgewise pitch & 1.079 & 1.172 & $8.62 \%$ \\
7 & $1^{\text {st }}$ blade edgewise yaw & 1.090 & 1.210 & $11.01 \%$ \\
8 & $2^{\text {nd }}$ blade flapwise yaw & 1.934 & 1.729 & $-10.60 \%$ \\
9 & $2^{\text {nd }}$ blade flapwise pitch & 1.922 & 1.970 & $2.50 \%$ \\
10 & $2^{\text {nd }}$ blade collective flap & 2.021 & 2.252 & $11.43 \%$ \\
11 & $2^{\text {nd }}$ tower side-to-side & 2.936 & 2.705 & $-7.87 \%$ \\
12 & $2^{\text {nd }}$ tower fore-aft & 2.900 & 2.855 & $-1.55 \%$ \\
\hline
\end{tabular}

\subsection{Soil springs}

206 As discussed above, the interaction between the monopile foundation and the surrounding soil may

207 significantly influence the dynamic behaviours of offshore wind turbine. To more accurately perform dynamic analysis, SSI is considered in the present study. Many different methods have been adopted 
by different researchers to consider SSI (e.g. $[6,12,26])$. Due to the simplicity and accuracy, the nonlinear soil springs are adopted in the present study.

211 In the nonlinear soil spring method, the lateral resistances of the soil against the foundation movements are depicted by the springs in the directions perpendicular and parallel to the rotor plane (the p-y springs in Fig. 4), and the vertical springs attached to the monopile are applied to simulate the shaft friction ( $t-z$ spring) and end bearing capacity at the tip of the monopile ( $Q-z$ spring). The space between each group of soil springs is selected as $1 \mathrm{~m}$ as suggested in [6], and they are modelled by the ground spring elements in ABAQUS. Fig. 4 shows the model of the monopile foundation attached with soil springs. The properties of the soil springs are described by the $p-y, t-z$ and $Q-z$ curves as recommended in API [34] and DNV-OS-J101 [35], which are briefly introduced in this section.

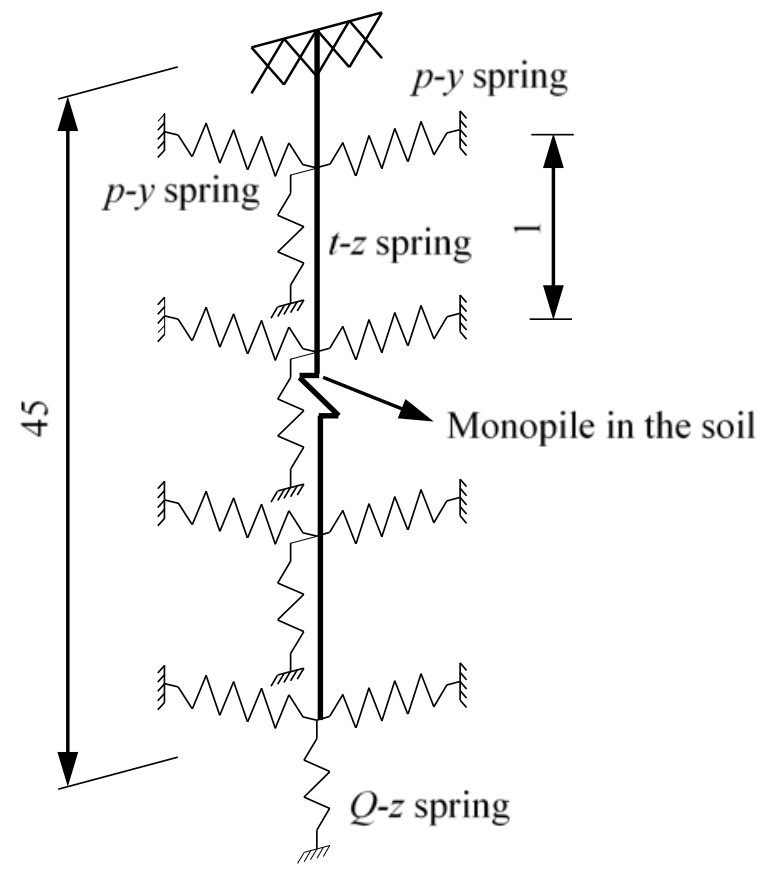

Fig. 4. SSI modelling (not to scale, dimensions in $\mathrm{m}$ )

In the present study, only the undrained clay is considered. As recommended in [34] and [35], the lateral force per unit length of the monopile $(p)$ is related to the undrained shear strength of soil $s_{\mathrm{u}}$ and the transition depth $Z_{\mathrm{R}}$ which is expressed by 


$$
Z_{R}=6 s_{u} d_{p} /\left(\gamma^{\prime} d_{p}+J s_{u}\right)
$$

226

227

where $d_{\mathrm{p}}$ is the outer diameter of the monopile; $\gamma^{\prime}$ is the effective unit weight of soil and it is $8 \mathrm{kN} / \mathrm{m}^{3}$ and $J$ is a dimensionless empirical constant, and the value of 0.5 is adopted in the present study [6]. When $s_{\mathrm{u}} \leq 100 \mathrm{kPa}$ and the depth below the sea bed $Z>Z_{\mathrm{R}}$, the lateral force per unit length of the monopile can be calculated as

$$
p= \begin{cases}0.5 p_{u}\left(y / y_{c}\right)^{1 / 3} & \text { for } y \leq 3 y_{c} \\ 0.72 p_{u} & \text { for } y>3 y_{c}\end{cases}
$$

where $y$ is the lateral displacement of the monopile and $y_{c}$ is the deformation at which the strength of the soil reaches one-half of the ultimate soil resistance, which is estimated as

$$
y_{c}=2.5 \varepsilon_{c} d_{p}
$$

in which, $\varepsilon_{\mathrm{c}}$ is the strain corresponding to one-half of the maximum stress in laboratory undrained compression tests of undisturbed soil. The relationship between $s_{\mathrm{u}}$ and $\varepsilon_{\mathrm{c}}$ proposed by Ashour et al. [36] is used in the present study. Three soil undrained shear strengths (25, 50 and $100 \mathrm{kPa}$ ) are considered in this study, the corresponding values of $\varepsilon_{\mathrm{c}}$ are $0.02,0.008$ and 0.006 respectively.

In Eq. (4), $p_{\mathrm{u}}$ is the ultimate lateral soil resistance per unit length of the monopile, which can be calculated as

$$
p_{u}=\left\{\begin{array}{cl}
\left(3 s_{u}+\gamma^{\prime} Z\right) d_{p}+J s_{u} Z & \text { for } 0<Z \leq Z_{R} \\
9 s_{u} d_{p} & \text { for } Z>Z_{R}
\end{array}\right.
$$

When $Z \leq Z_{\mathrm{R}}$, $p$ becomes

$$
p=\left\{\begin{array}{cl}
0.5 p_{u}\left(y / y_{c}\right)^{1 / 3} & \text { for } y \leq 3 y_{c} \\
0.72 p_{u}\left(1-\left(1-Z / Z_{R}\right)\left(y-3 y_{c}\right) / 12 y_{c}\right) & \text { for } 3 y_{c}<y \leq 15 y_{c} \\
0.72 p_{u}\left(Z / Z_{R}\right) & \text { for } y>15 y_{c}
\end{array}\right.
$$

The axial resistance of the soil is modelled by a combination of shaft friction and end bearing capacity at the monopile tip as shown in Fig. 4. The relationship between the mobilized soil-monopile shear transfer and monopile displacement at any depth can be represented by the $t$-z curve [34]. Similarly, Q-z curve is used to describe the relationship between the end bearing resistance and axial tip deflection [34]. In API [34], the $t / t_{\max }$ versus $z / z_{\text {peak }}$ and $Q / Q_{\mathrm{p}}$ versus $z / d_{\mathrm{p}}$ relationships are tabulated. In which $z$ is the monopile axial deflection at any depth below the sea bed; $z_{\text {peak }}$ is the displacement corresponding to the maximum soil-monopile adhesion and the value of $z_{\text {peak }}$ is typically $1 \%$ of the 
monopile outer diameter $d_{\mathrm{p}} ; t$ and $t_{\max }$ are the mobilized and maximum soil-monopile adhesion respectively; $Q$ and $Q_{\mathrm{p}}$ are the mobilized and end bearing capacities respectively. The values of $t_{\max }$ and $Q_{\mathrm{p}}$ are dependent on the undrained soil shear strength $s_{\mathrm{u}}$ and can be calculated as suggested in [34]. Substituting $t_{\mathrm{max}}, Z_{\mathrm{peak}}, Q_{\mathrm{p}}$ and $d_{\mathrm{p}}$ into the tables in [34], the $t$ versus $z$ and $Q$ versus $z$ relationships can be obtained.

251

Fig. 5 shows the $p-y, t-z$ and $Q-z$ curves at different depths (5 to $45 \mathrm{~m}$ with an increment of $10 \mathrm{~m}$ ) below the sea bed with $s_{\mathrm{u}}=50 \mathrm{kPa}$ (corresponds to a typical medium clay). As shown in Fig. 5(a), $p$ is not influenced by the depth when it is larger than $25 \mathrm{~m}$. Fig. 5 (c) shows the relationship at the monopile tip, the depth is fixed and therefore only one curve is included in Fig. 5 (c).

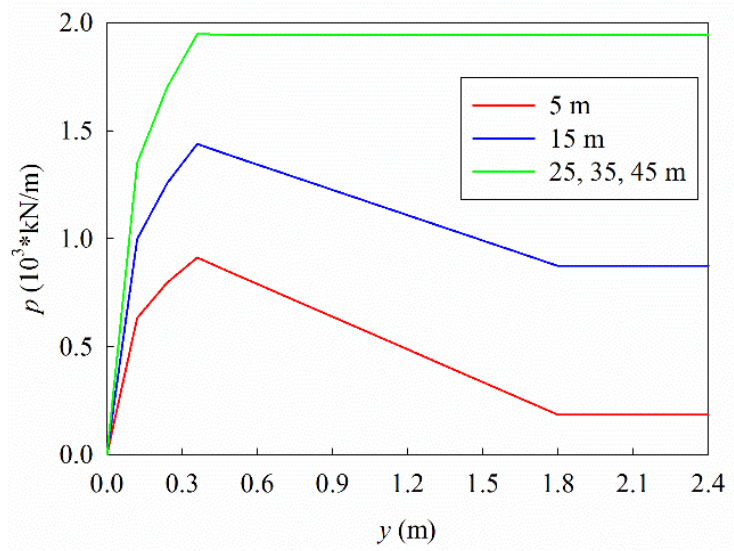

(a) $p-y$ curve

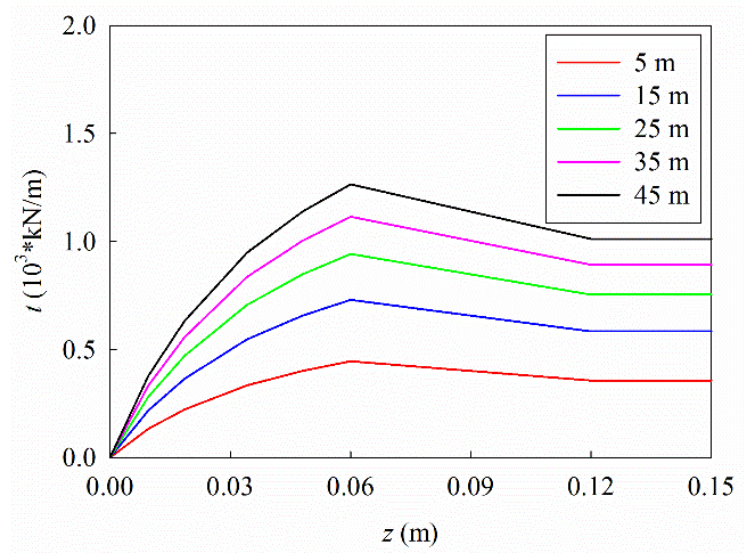

(b) $t$-z curve

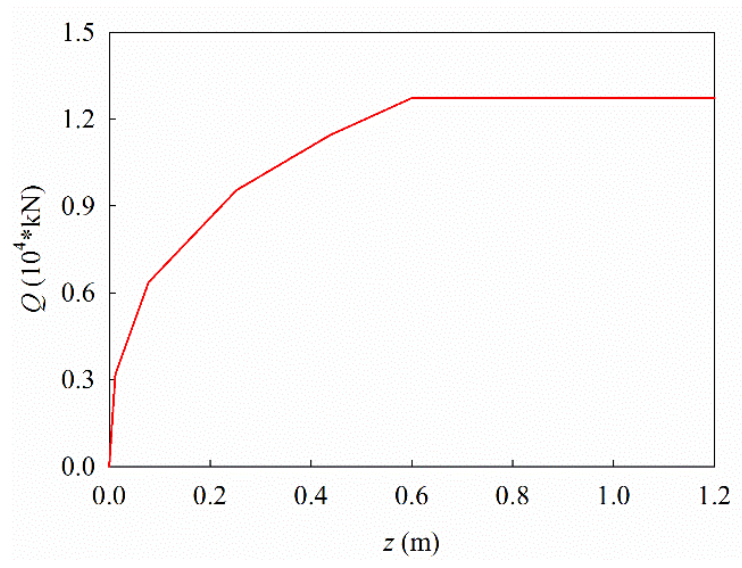

(c) Q-z curve 


\subsection{Vibration characteristics}

259 After the nonlinear soil springs are defined, the vibration frequencies and vibration modes of the wind 260 turbine with the consideration of SSI are calculated. As discussed above, three undrained shear 261 strengths of soil are considered in the present study. For conciseness, only the results when $s_{\mathrm{u}}=50 \mathrm{kPa}$

262 are reported. Table 4 tabulates the first ten natural frequencies and Fig. 6 shows the corresponding vibration modes. To show the mode shape more clearly, the blades are not shown when the mode is dominated by the tower vibration.

\section{Table 4}

267 First ten natural frequencies of the wind turbine with SSI $\left(s_{\mathrm{u}}=50 \mathrm{kPa}\right)$

\begin{tabular}{ccc}
\hline Mode & Description & $f(\mathrm{~Hz})$ \\
\hline 1 & tower fore-aft $\left(1^{\text {st }}\right.$ order $)$ & 0.154 \\
2 & tower side-to-side $\left(1^{\text {st }}\right.$ order $)$ & 0.156 \\
3 & blade flapwise yaw $\left(1^{\text {st }}\right.$ order $)$ & 0.474 \\
4 & blade flapwise pitch $\left(1^{\text {st }}\right.$ order $)$ & 0.536 \\
5 & blade collective flap $\left(1^{\text {st }}\right.$ order $)$ & 0.596 \\
6 & tower side-to-side $\left(2^{\text {nd }}\right.$ order $)$ & 1.096 \\
7 & tower fore-aft $\left(2^{\text {nd }}\right.$ order $)$ & 1.121 \\
8 & blade edgewise pitch $\left(1^{\text {st }}\right.$ order $)$ & 1.206 \\
9 & blade edgewise yaw $\left(1^{\text {st }}\right.$ order $)$ & 1.250 \\
10 & blade flapwise yaw $\left(2^{\text {nd }}\right.$ order $)$ & 1.626 \\
\hline
\end{tabular}

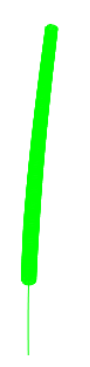

Mode 1

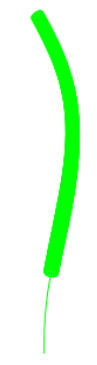

Mode 6

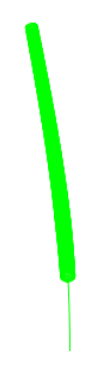

Mode 2

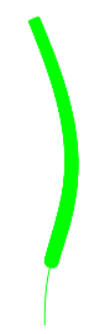

Mode 7

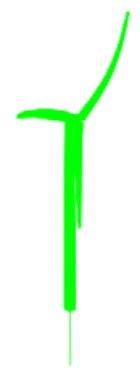

Mode 3

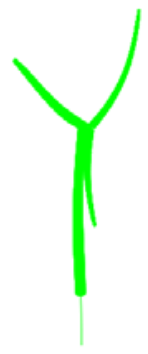

Mode 8

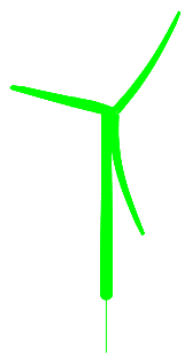

Mode 4

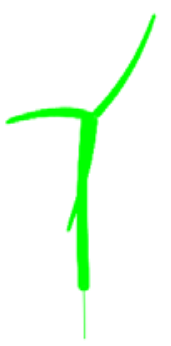

Mode 9

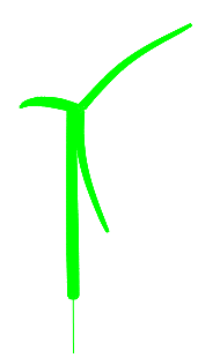

Mode 5

Fig. 6. Vibration modes of the wind turbine with SSI 
271 The damping mechanism of offshore wind turbine is quite complicated and it normally comprises

272 structural damping, aerodynamic damping, hydrodynamic damping and soil damping, which account for the contributions of the structure itself, wind, surrounding sea water and supporting soil respectively [25]. The structural damping ratios of the blades and the tower are $0.48 \%$ and $1 \%$ respectively as suggested in [1]. Aerodynamic damping results from the relative velocity between the wind and the rotating blades, which depends on the wind velocity, rotor speed, the geometrical configurations of the blades and the flow around the blades [37]. It is not easy to accurately obtain the aerodynamic damping. The aerodynamic damping in the fore-aft direction for an operating wind turbine is normally within the range of $1-6 \%$ [38]. Without loss of generality, a constant value of $3.5 \%$ is adopted in the present study as suggested by Bisoi and Haldar [6, 7]. For a parked wind turbine or in the side-to-side direction, previous studies (e.g. [25, 38]) revealed that the aerodynamic damping ratio is almost zero, and zero is adopted in the present study. The hydrodynamic damping results from the drag between the tower and the surrounding water. The upper limit of hydrodynamic damping ratio is about $0.23 \%$ [25] and this value is used in this study. The soil damping develops from SSI and it includes the material damping of the soil and the wave radiation damping which may go up to as high as $20 \%$ for the subgrade medium in some cases [6]. However, as will be demonstrated in Sections 3.1 and 3.3, the frequency contents of wind and sea wave loads are very low (up to 0.2 and $0.8 \mathrm{~Hz}$ respectively), and as indicated in [39] very little energy is dissipated by the radiation of waves when the excitation frequency is below $1 \mathrm{~Hz}$, the wave radiation damping is therefore neglected in the numerical model. The material damping is thus dominant in the soil damping and $1 \%$ is assumed in the present study [25]. Therefore, summing all the components together, the damping ratio is $3.98 \%$ in the fore-aft direction for the rotating blades; for the parked blades or in the side-to-side direction, the value is $0.48 \%$; and the damping ratio of the tower taking into account SSI is $2.23 \%$. The damping of offshore wind turbine is considered by means of Rayleigh damping and the first out-of-plane and in-plane vibration frequencies of the tower and blades are used to calculate the mass and stiffness coefficients for the tower and blades respectively [40]. 


\section{Vibration sources}

299 In the present study, the wind and sea wave loadings, which are experienced during the whole lifetime of an offshore wind turbine, are considered as the external vibration sources. The wind and sea wave loads are stochastically simulated based on the sophisticated simulation techniques, and they are briefly introduced in this section for completeness of the paper.

303

\subsection{Wind load on the tower}

The wind load can be decomposed into a constant mean wind load and a fluctuating component. The

Kaimal spectrum [10] is used to model the power spectral density (PSD) function of the fluctuating

wind velocity along the tower, which is given by

$$
S_{v v}(h, f)=\frac{v_{*}^{2}}{f} \frac{200 c}{(1+50 c)^{5 / 3}}
$$

where

$$
c=f h / \bar{v}(h)
$$

and

$$
\bar{v}(h)=v_{*} \ln \left(h / z_{0}\right) / K
$$

in which $h$ is the height of the location where wind load is calculated; $f$ is the frequency in $\mathrm{Hz} ; \bar{v}$ is the mean wind velocity; $v *$ is the friction velocity; $c$ is the Monin coordinate; $K$ is the von-Karman's constant, which is generally taken as 0.4 [41] and $z_{0}$ is the roughness length.

For a continuous line-like structure, like the tower, the wind loads at different locations along the tower are different but with certain similarities, which is known as the spatial correlation effect. The spatial correlation effect is normally described by a spatial coherency loss function. The modal fluctuating drag force power spectrum, which considers the influence of spatial correlation effect, can be calculated by [10]

$$
S_{f, j}(f)=\left(C_{d t} A \rho\right)^{2} \sum_{k=1}^{N} \sum_{l=1}^{N} S_{v_{k} v_{l}}(f) \bar{v}_{k} \bar{v}_{l} \phi_{j}(k) \phi_{j}(l)
$$
in which $C_{\mathrm{dt}}$ is the drag coefficient of the tower; $A$ is the total area of the tower exposed to the wind; $\rho$ is the air density; $\bar{v}_{k}$ and $\bar{v}_{l}$ are the mean wind velocities at locations $k$ and $l$ respectively; $\phi_{j}(k)$ and $\phi_{j}(l)$ 
are the $j$ th mode shape at locations $k$ and $l$. As will be demonstrated in the following analysis, the energy of wind load mainly concentrates in the low frequency range (see Fig. 7) and normally the first vibration mode of the tower can be excited by the wind, $j=1$ is therefore used in the simulation. $S_{v k v l}$ is the cross PSD function of wind velocity between locations $k$ and $l$, which can be expressed as

$$
S_{v_{k} v_{l}}(f)=\sqrt{S_{v_{k} v_{k}}(f) S_{v_{l} v_{l}}(f)} \operatorname{coh}(k, l ; f)
$$

where $S_{v k v k}$ and $S_{v l v l}$ are the wind velocity auto PSDs as given by Eq. (8); $\operatorname{coh}(k, l ; f)$ is the spatial coherency loss function between locations $k$ and $l$ and the model as proposed by Huang et al. [42] is adopted in the present study

$$
\operatorname{coh}(k, l ; f)=\exp \left(-\frac{D \omega|k-l|}{2 \pi \hat{v}}\right) \exp \left(-i \frac{k-l}{v_{a p p}} \omega\right) \quad(k>l)
$$

in which, $|k-l|$ and $\hat{v}$ are the distance and average mean wind velocity between locations $k$ and $l$ respectively; $D$ is a decay constant; $\omega$ is angular frequency in $\mathrm{rad} / \mathrm{s}$ and $v_{\text {app }}$ is the apparent wave velocity. For $k<l$, the spatial coherency loss function is the complex conjugate of that with $k>l$.

The time histories of the fluctuating drag force with zero mean then can be simulated by using the Inverse Fast Fourier transform (IFFT) technique (e.g. [43, 44]).

The mean wind drag force can be calculated by

$$
\bar{f}_{\text {mean }, i}=\frac{1}{2} C_{d t} A_{i} \rho \bar{v}_{i}^{2}
$$

in which, $A_{i}$ and $\bar{v}_{i}$ are the area associated with location $i$ and the mean wind velocity at location $i$ respectively.

The wind loads at different locations along the tower are different. To simplify the analysis, the tower is divided into nine segments in the simulation and the drag force is assumed to be the same within each segment. The lengths of the top and bottom segments are 5 and $15 \mathrm{~m}$ respectively and other segments are $10 \mathrm{~m}$ length. The mean wind velocity at the top of the tower is taken as $15 \mathrm{~m} / \mathrm{s}$, and the roughness length, air density, drag coefficient, decay constant and apparent wave velocity are 0.005, $1.2 \mathrm{~kg} / \mathrm{m}^{3}, 1.2,0.04$ and $10 \mathrm{~m} / \mathrm{s}$ respectively. Fig. 7 shows the fluctuating wind velocity PSDs in segments S1 (85-90 $\mathrm{m}$ along the tower) and S5 (45-55 m) and the corresponding model values. The model and simulated coherency loss functions between segments S1and S5 are presented in Fig. 8. As 

example segments (S1 and S5) are shown in Fig. 9.

346
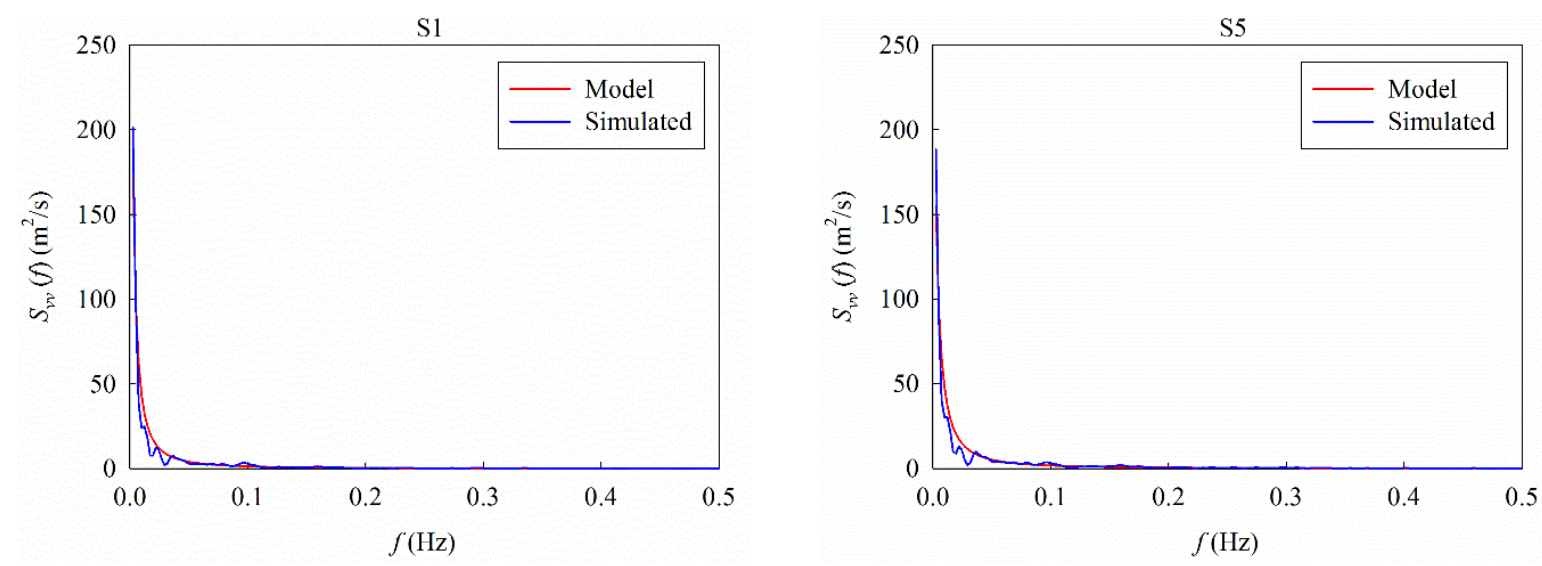

Fig. 7. Comparisons of the simulated wind velocity with the model PSDs

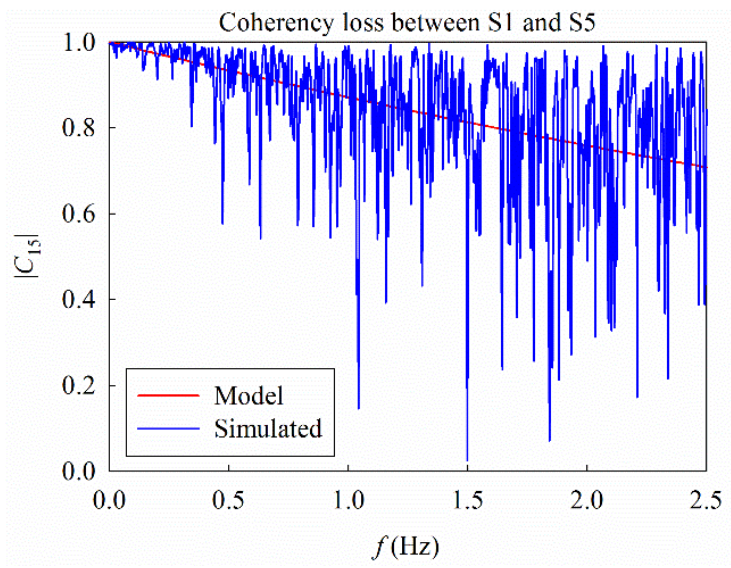

Fig. 8. Comparison between the simulated and model coherency loss functions
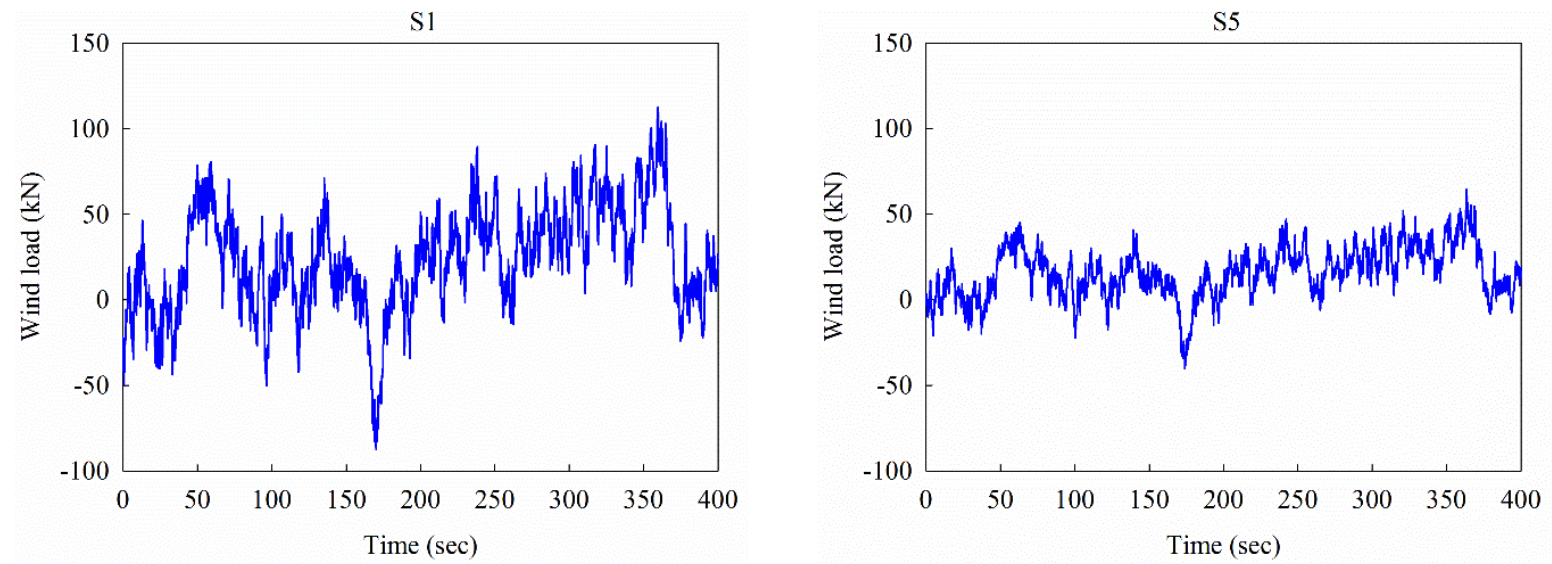

Fig. 9. Wind loads in segments S1 and S5 


\subsection{Wind load on the blades}

352 The wind loads on the blades are influenced by the wind velocity, rotor velocity, pitch angle, the number of blades and geometrical configurations of the blade (e.g. blade profile, twist and chord distribution) [16]. To more realistically estimate the wind loads on the rotating blades, the blade element momentum (BEM) method, which couples the momentum theory with the local events taking place at the actual blades [9], is adopted in the present study. In the BEM method, it is assumed that all sections are independent along the rotor, i.e. no aerodynamic interaction between different sections. Each blade therefore can be divided into several elements and wind load on each element can be calculated separately [9].

The relative wind velocity on each element of the blade, $v_{\text {rel, }}$ is given by

$$
v_{r e l}\left(r, t_{s}\right)=\sqrt{\left(\bar{v}\left(r, t_{s}\right)(1-a)+v_{f}\left(t_{s}\right)\right)^{2}+\left(\Omega r\left(1+a^{\prime}\right)\right)^{2}}
$$

361

where $r$ is a radial distance of the element from the centre of the hub; $t_{s}$ is time; $a$ and $a$ are the axial and tangential induction factors respectively; $\Omega$ is the rotor velocity in $\mathrm{rad} / \mathrm{s}$; $\bar{v}$ is the mean wind velocity and $v_{\mathrm{f}}$ is the fluctuating wind velocity, the calculations of which are discussed below.

The height of each blade element $h\left(r, t_{s}\right)$ experiences a sinusoidal variation in magnitude as the rotation of the blades and the frequency of this variation is the same as the rotor frequency. With the definition of blade numbers in Fig. 2, $h\left(r, t_{s}\right)$ can be expressed as

$$
\begin{gathered}
h\left(r, t_{s}\right)=H_{h u b}+r \cos \left(\Omega t_{s}+\theta_{i}\right) \\
\theta_{i}=\pi-\frac{2 \pi}{3}(i-1) \quad i=1,2,3
\end{gathered}
$$

where $H_{\text {hub }}$ is the hub height and $\theta_{i}$ is the phase difference between blades. By submitting $h\left(r, t_{s}\right)$ into Eq. (10), the mean wind velocity $\bar{v}$ in Eq. (15) therefore can be obtained.

Due to the rotation of the blades, the PSD of the fluctuating wind velocity $v_{\mathrm{f}}$ is not a constant but varies with time, namely it is a time-variant rotational sampled spectrum. However, not to further complicate the problem, an isotropic, homogeneous turbulence at the hub height is assumed to represent the turbulence over the rotor field in the present study as suggested by many previous 
studies (e.g. [16]). Based on this assumption, the fluctuating wind velocity $v_{\mathrm{f}}$ in Eq. (15) can be estimated by using the PSD of wind velocity at the hub height defined in Eq. (8).

After the relative wind velocity is determined (Eq. (15)), the local lift and drag forces on each element can be calculated as follows [9]

$$
\begin{aligned}
& p_{l}\left(r, t_{s}\right)=\frac{1}{2} \rho v_{r e l}^{2}\left(r, t_{s}\right) l(r) C_{l b}(\alpha) \\
& p_{d}\left(r, t_{s}\right)=\frac{1}{2} \rho v_{r e l}^{2}\left(r, t_{s}\right) l(r) C_{d b}(\alpha)
\end{aligned}
$$

377 in which, $l$ is the chord length; $C_{\mathrm{lb}}$ and $C_{\mathrm{db}}$ are the lift and drag coefficients of the blade respectively and it is related to the local angle of attack, which is defined by

$$
\alpha\left(r, t_{s}\right)=\varphi\left(r, t_{s}\right)-\beta\left(t_{s}\right)-\kappa(r)
$$

379

380

381

382

383

where $\beta$ is the pitch angle and it is $10^{\circ}$ in the present study and $\kappa$ is the pre-twist angle of each element with respect to the hub, which decreases from the bottom of $13.3^{\circ}$ to the tip of $0^{\circ}[1] . \varphi\left(r, t_{s}\right)$ is the flow angle and it can be calculated as

$$
\varphi\left(r, t_{s}\right)=\tan ^{-1}\left(\frac{(1-a) \bar{v}\left(r, t_{s}\right)+v_{f}\left(t_{s}\right)}{\left(1+a^{\prime}\right) \Omega r}\right)
$$

Fig. 10 shows the lift and drag coefficients of the blade with respect to the angle of attack.

The local wind loads in the directions parallel and perpendicular to the rotor plane therefore can be calculated by projecting the local lift and drag forces along the edgewise and flapwise directions respectively as shown in Fig. 11, which can be expressed as

$$
\left\{\begin{array}{l}
p_{t}\left(r, t_{s}\right) \\
p_{n}\left(r, t_{s}\right)
\end{array}\right\}=\left[\begin{array}{cc}
\sin \left(\varphi\left(r, t_{s}\right)\right) & -\cos \left(\varphi\left(r, t_{s}\right)\right) \\
\cos \left(\varphi\left(r, t_{s}\right)\right) & \sin \left(\varphi\left(r, t_{s}\right)\right)
\end{array}\right]\left\{\begin{array}{c}
p_{l}\left(r, t_{s}\right) \\
p_{d}\left(r, t_{s}\right)
\end{array}\right\}
$$

The total in-plane and out-of-plane wind loads on the blade then can be obtained by integrating the wind loads on each blade element over the entire rotor length as

$$
\begin{aligned}
& F_{t}\left(t_{s}\right)=\int_{0}^{R} p_{t}\left(r, t_{s}\right) \phi_{e, 1}(r) d r \\
& F_{n}\left(t_{s}\right)=\int_{0}^{R} p_{n}\left(r, t_{s}\right) \phi_{f, 1}(r) d r
\end{aligned}
$$



analysis and they are shown in Fig. 12.
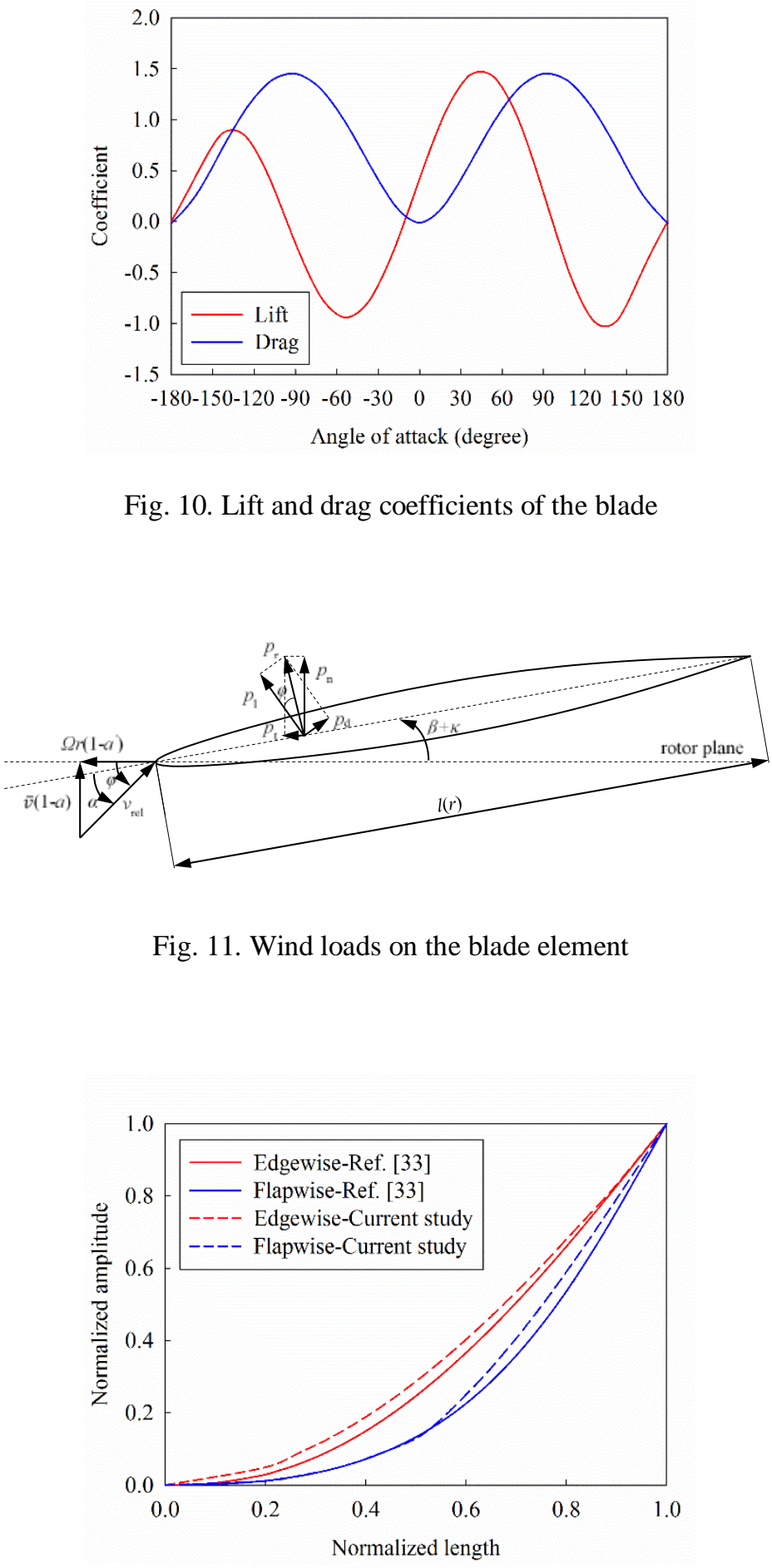

Fig. 12. First edgewise and flapwise vibration mode shapes of the blade 
401

402

403

404

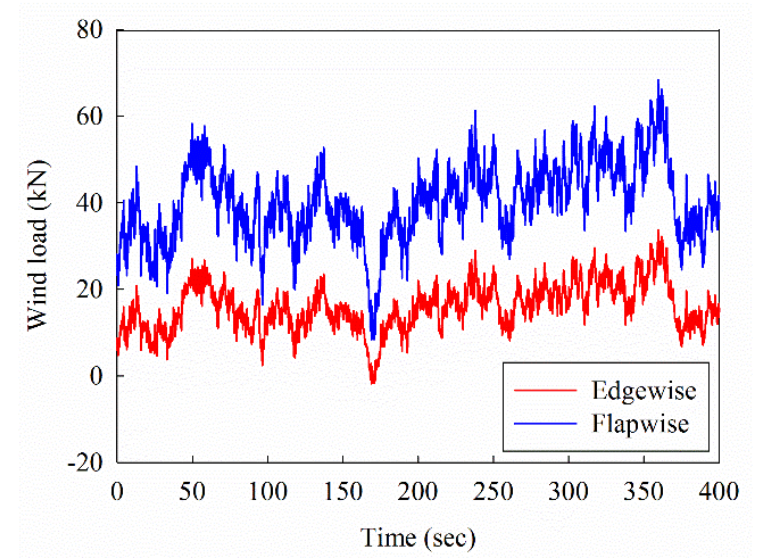

(b) Operating condition
405

406

407

408

409

410

411

412

413

414

415

416

417

418

419

420

Based on the BEM method discussed above, the total in-plane and out-of-plane wind loads of a rotating blade can be calculated. When the parked condition is of interest, the same procedure can be followed, and the above equations can be simplified by submitting $\Omega=0$ into Eqs. (15), (16), and (21).

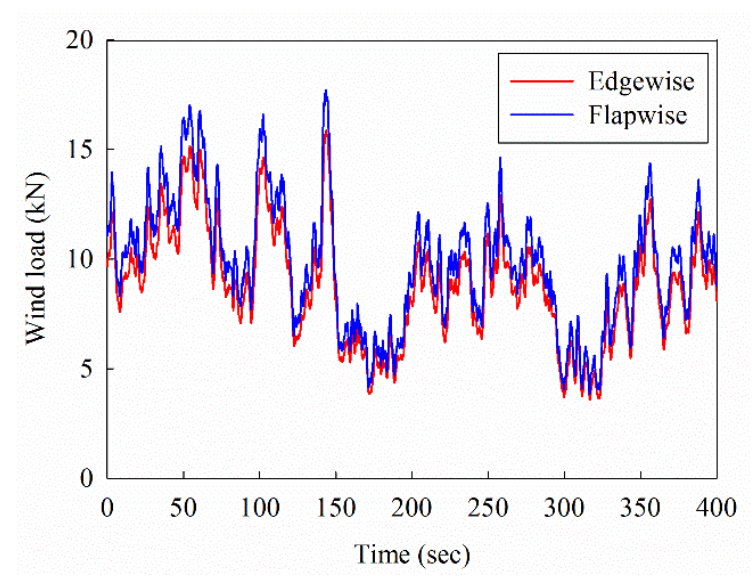

(a) Parked condition

Fig. 13. Edgewise and flapwise wind loads on Blade 2

The wind loads on each blade of the wind turbine when it is in the parked and operating conditions therefore can be simulated and Fig. 13 shows the edgewise and flapwise wind loads on Blade 2 under different conditions. In this simulation, the mean wind velocity at the tower top is $15 \mathrm{~m} / \mathrm{s}$ and the rotor speed is 12.1 rounds per minute. For conciseness, the wind loads on other two blades are not shown. As shown in Fig. 13(a), the flapwise wind loads are slightly larger than the edgewise wind loads when the wind turbine is in the parked condition, this is because the flow angle is a constant and equals to $\pi / 2$ based on Eq. (21) when $\Omega=0$ and the angle of attack is between 67 and 80 degrees, therefore the lift and drag coefficients have a small difference as shown in Fig. 10, which in turn lead to nonsignificant difference between the flapwise and edgewise wind loads on the parked blades. When the wind turbine is in the operating condition, the wind loads in the edgewise and flapwise directions are obviously different and the wind load in the flapwsie direction is larger than that in the edgewise direction. Moreover, comparing Fig. 13(a) with 13(b), it is obvious that the wind loads on the rotating blades are much larger than those on the parked blades since the relative wind velocity becomes larger based on Eq. (15) due to the rotation of the blades. 


\subsection{Sea wave load on the monopile}

422 To calculate the sea wave load acting on the monopile, the JONSWAP spectrum, which has the 423 following form [45] is used to simulate the sea surface elevation

$$
S_{\eta \eta}(f)=\alpha_{P} g^{2}(2 \pi)^{-4} f^{-5} \exp \left[-\frac{5}{4}\left(\frac{f_{m}}{f}\right)^{4}\right] \gamma^{\exp \left[-\frac{\left(f-f_{m}\right)^{2}}{2 \sigma^{2} f_{m}^{2}}\right]}
$$

424 where $\eta$ is the sea surface elevation; $g$ is the gravitational acceleration; $\gamma$ is the peak enhancement 425 factor (typically 3.3 [4]) and $f$ is frequency in Hz. $\alpha_{\mathrm{P}}, f_{m}$ and $\sigma$ are three constants, which are [4]

$$
\begin{aligned}
& \alpha_{P}=0.076\left(F g / v_{10}^{2}\right)^{-0.22} \\
& f_{m}=11\left(v_{10} F / g^{2}\right)^{-1 / 3} / \pi
\end{aligned}
$$

426 and

$$
\sigma= \begin{cases}0.07 & f \leq f_{m} \\ 0.09 & f>f_{m}\end{cases}
$$

427 in which $v_{10}$ is the mean wind velocity at $10 \mathrm{~m}$ above sea surface and $F$ is the fetch length.

428 The sea surface elevation $\eta\left(t_{s}\right)$ in the time domain then can be simulated as

$$
\eta\left(t_{s}\right)=\sum_{i=1}^{n} \sqrt{2 d \omega S_{\eta \eta}\left(\omega_{i}\right)} \cos \left(\omega_{i} t_{s}+\Phi\left(\omega_{i}\right)\right)
$$

where $\Phi$ is the random phase angle uniformly distributed over the range of $[0,2 \pi]$.

430 The velocity and acceleration of water particles in the horizontal direction can be expressed as [46]

$$
\begin{aligned}
& v_{x}=\frac{H \omega}{2} \frac{\cosh k_{w}\left(d_{w}+z_{w}\right)}{\sinh k_{w} d_{w}} \cos \left(k_{w} x_{w}-\omega t_{s}+\varphi\right) \\
& a_{x}=\frac{H \omega^{2}}{2} \frac{\cosh k_{w}\left(d_{w}+z_{w}\right)}{\sinh k_{w} d_{w}} \sin \left(k_{w} x_{w}-\omega t_{s}+\varphi\right)
\end{aligned}
$$

431 in which $x_{\mathrm{w}}$ and $z_{\mathrm{w}}$ denote the horizontal and vertical coordinates respectively; $d_{\mathrm{w}}$ is water depth, which is $20 \mathrm{~m}$ in the present study; $H$ is the wave height, which is two times of the amplitude of the sea surface elevation; $\omega$ is angular frequency in $\mathrm{rad} / \mathrm{s}$ and $k_{\mathrm{w}}$ is the sea wave number, which can be estimated based on the following equation [46]

$$
\omega^{2}=g k_{w} \tanh \left(k_{w} d_{w}\right)
$$

435 In the present study, the following parameters are used: $g=9.8 \mathrm{~m} / \mathrm{s}^{2}, v_{10}=11.5 \mathrm{~m} / \mathrm{s}$ and $F=40,000 \mathrm{~m}$.

436 The peak wave frequency $f_{\mathrm{m}}$ is therefore $0.208 \mathrm{~Hz}$ based on Eq. (27) and the wave period is $T=1 / f_{\mathrm{m}}=$ 
$4374.81 \mathrm{~s}$. The wave length can be calculated as $\lambda=g T^{2} / 2 \pi=36 \mathrm{~m}$ [46], and it is larger than five times of 438 the diameter of the monopile. Based on the specifications in [32], the Morison formula can be used to 439 calculate the sea wave load. According to the Morison equation, the transverse sea wave load per unit 440 length of the monopile can be calculated as

$$
\begin{gathered}
F_{w}=\frac{1}{2} \rho_{w} C_{d p} d_{p}\left|v_{x}\right| v_{x}+\rho_{w} C_{m} A_{p} a_{x} \\
C_{m}=C_{a}+1
\end{gathered}
$$

441 where $C_{\mathrm{dp}}, C_{\mathrm{m}}$ are the drag and inertia coefficients respectively, and $C_{\mathrm{dp}}=1.2, C_{\mathrm{m}}=2.0$ are adopted in 442 the simulation. It should be noted that the first term represents the contribution of the quadratic drag 443 force, and the second term is the inertia force.

444 In the simulation, the monopile in the water is divided into two segments and the length of each segment is $10 \mathrm{~m}$. Fig. 14 shows the PSDs of the simulated sea surface elevation and the given model, good match is observed. Fig. 15 shows the simulated sea wave load time history at the mean sea level; the sea wave loads at other locations along the monopile are not shown for conciseness. As mentioned above, the blade, tower and monopile in the sea water are divided into a few segments. In the FE model, a reference point is defined in each segment and coupled with the cross section of the corresponding segment, the simulated wind and sea wave loadings are applied on these reference points.

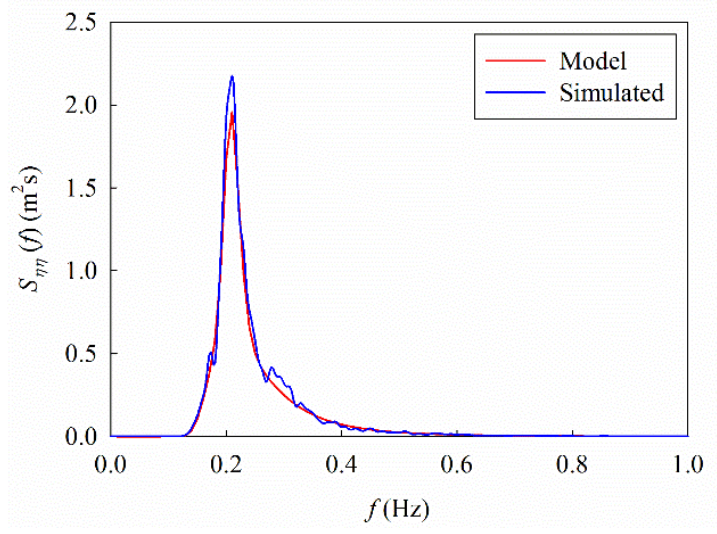




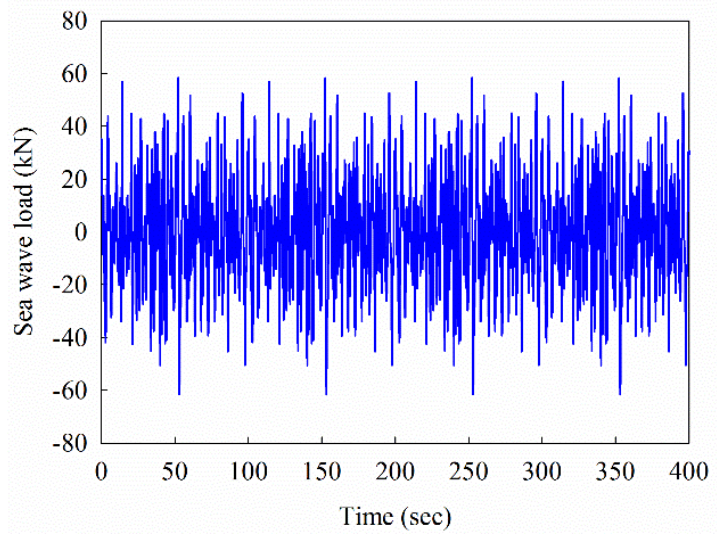

Fig. 15. Sea wave load time history at mean sea level

457

\section{Numerical results}

\subsection{Influence of operational conditions}

460 To examine the influence of operational conditions of wind turbine on the structural responses, two cases are investigated in this section. In the first case, the wind turbine is in the parked condition, with the locations of the blades shown in Fig. 2(a). In the second case, the blades are rotating with a uniform angular velocity of $\Omega=1.27 \mathrm{rad} / \mathrm{s}$, which corresponds to the rated rotor speed of NREL $5 \mathrm{MW}$ wind turbine (12.1 rpm). The wind and sea wave loads shown in Figs. 9 and 15 are applied to the tower respectively in both cases. For the wind loads on the blades, they depend on the rotor velocity as discussed in Section 3.2. Different wind loads as shown in Fig. 13 are applied in different cases. In both cases, a duration of $400 \mathrm{~s}$ is considered for all the external vibration sources. Not to further complicate the problem, SSI is not considered in this section, i.e. the wind turbine is fixed at the sea bed level.

It is obvious that the responses along the tower are different and the maximum responses occur at the tower top. For conciseness, only the maximum responses are discussed in the present study. Fig. 16 shows the displacement time histories at the top of the tower in the fore-aft and side-to-side directions. The red curves are the results when the blades are in the parked condition, and the blue curves are the displacements when they are rotating. It shows that the displacements in the operating condition are larger than those in the parked condition in both directions. As shown in Fig. 16(a), the maximum fore-aft displacement at the top of the tower is $0.473 \mathrm{~m}$ occurring at $t=171 \mathrm{~s}$ when the wind turbine is 
still. When the blades are rotating, a larger maximum displacement of $0.674 \mathrm{~m}$ occurs at $t=362 \mathrm{~s}$. For

478 the side-to-side displacement at the top of the tower, the maximum values are 0.093 and $0.206 \mathrm{~m}$

479 respectively when the wind turbine is in the parked and operating conditions (Fig. 16(b)). These

480

481

482

483

484

485

486

487

488

489

490

491

492

493

494

495

496

results are actually expected since as shown in Fig. 13, when the blades are rotating, the wind loads acting on the blades are larger compared to the parked condition. Larger wind loads on the blades result in more severe interaction between the tower and blades and therefore larger tower responses. These results indicate that previous studies by assuming the wind turbines in the parked condition may result in non-conservative structural response estimations, which in turn may lead to the unsafe design of structural components.

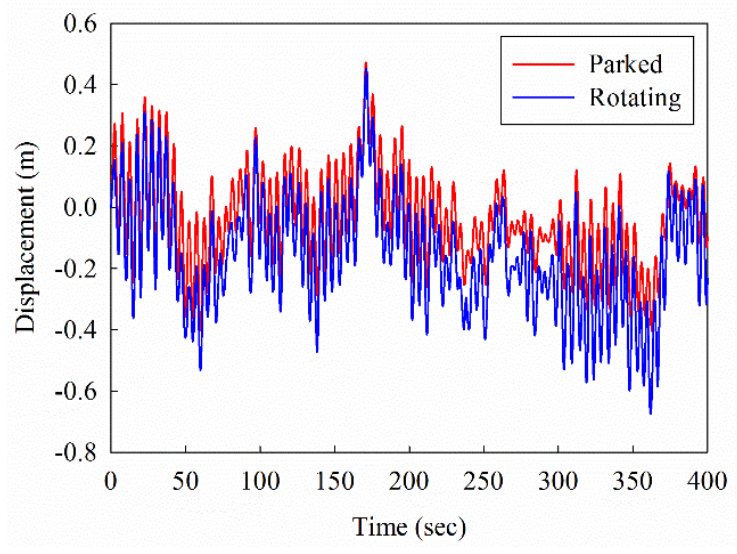

(a) Fore-aft

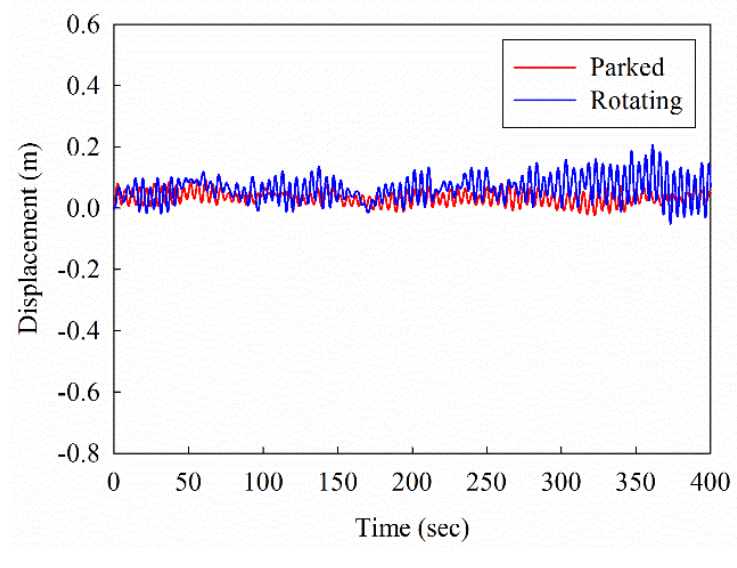

(b) Side-to-side

Fig. 16. Fore-aft and side-to-side displacement time histories at the tower top

Comparing Fig. 16(a) with 16(b), it is obvious that the side-to-side displacements of the tower are much smaller than the fore-aft responses in both cases. Two reasons lead to these results. The first one is that the wind and sea wave loads are only applied in the fore-aft direction on the tower in the numerical simulation, and no external vibration sources are acted in the side-to-side direction. The other reason is that as shown in Fig. 13, the wind loads on the blades in the flapwise (fore-aft direction corresponds to tower) and edgewise (side-to-side) directions are almost the same when the wind turbine is in the parked condition (Fig. 13(a)), while when it is in the operating condition, the wind loads in the flapwise direction are much larger than those in the edgewise direction (Fig. 13(b)). The 
larger wind loads on the blades result in more severe interaction between the tower and blades, and lead to the larger tower responses in the fore-aft direction as discussed above.

Fig. 17 shows the PSDs of the acceleration responses at the top of the tower in the fore-aft and sideto-side directions when the blades are in the parked and operating conditions. To more clearly obtain the dominant frequencies of the structural responses, an N/4-point Hamming window is used to smooth the PSDs in the present study, in which $N$ is the number of the data to be analysed. As shown in Fig. 17(a), an obvious peak appears at $0.204 \mathrm{~Hz}$. As shown in Table 5 in Section 4.2, this value corresponds to the first vibration mode of the tower in the fore-aft direction, which means the first vibration mode is excited by the external vibration sources. Fig. 17 also shows that another peak occurs at 0.603 and $0.647 \mathrm{~Hz}$ respectively when the wind turbine is in the parked and operating conditions. As shown in Table 5, $0.603 \mathrm{~Hz}$ is the first collective flap vibration mode of the blades when the wind turbine is in the parked condition. For $0.647 \mathrm{~Hz}$, this frequency cannot be directly found in Table 5, this is because the rotating condition cannot be directly considered in the modal analysis by using ABAQUS. This frequency corresponds to the first collective flap vibration mode of the blades when the wind turbine is in the operating condition. These results indicate again that the interaction between the tower and blades makes the vibrations of the blades contribute to the tower responses. Compared to the parked condition, the first collective flap vibration frequency of the blades is slightly larger when it is in the operating condition as shown in Fig. 17(a). This is because the centrifugal stiffness of the blades is generated when the wind turbine is operating, which in turn leads to large structural stiffness and vibration frequency of the blades. For the PSDs in the side-to-

517 side direction, Fig. 17(b) shows that only one peak appears at $0.208 \mathrm{~Hz}$, and this peak corresponds to 518 the first vibration mode of the tower in the side-to-side direction as shown in Table 5. Comparing the results in Fig. 17(b) with those in Fig. 17(a), it is obvious that the energies are much smaller in the side-to-side direction, which results in the smaller tower responses in this direction as shown in Fig. 16. Similarly, compared to the red curve, the values in the blue curve are larger, and this explains the larger structural responses in the operating condition as shown in Fig. 16. 


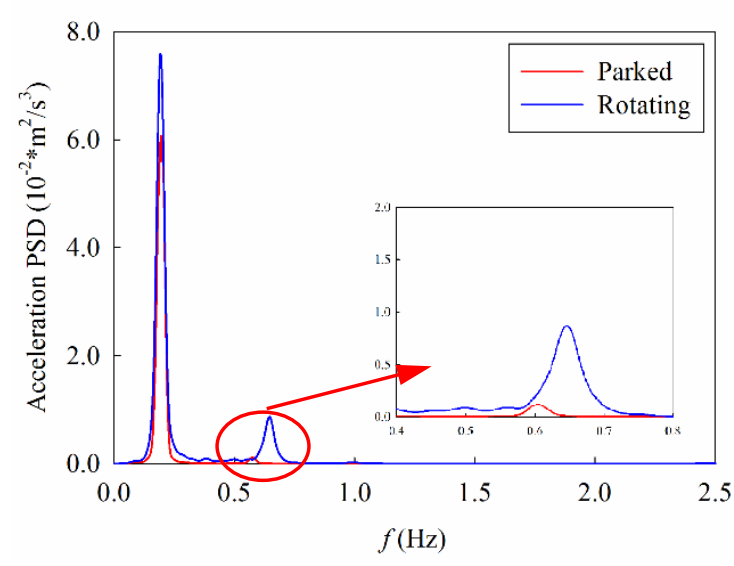

(a) Fore-aft

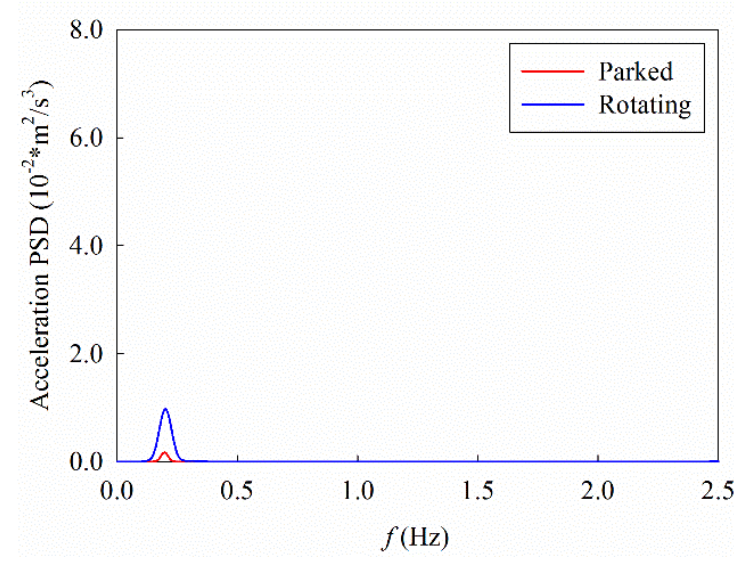

(b) Side-to-side

Fig. 17. Fore-aft and side-to-side acceleration PSDs at the tower top

Fig. 18 shows the displacement (relative to the tower top) time histories at the tips of blades in the flapwise direction. Wind load acting on the blade can be decomposed into a constant mean and a fluctuating component as discussed in Section 3. The mean value of the fluctuating term is zero. However, the total wind load on the blade has a non-zero mean because of the constant component of the wind pressure as shown in Fig. 13, which results in the non-zero baseline of the structural responses shown in Fig. 18. As shown, the maximum flapwise displacements at the tips of Blades 1, 2 and 3 are $0.429,0.544,0.544 \mathrm{~m}$ respectively when the wind turbine is in the parked condition. The displacements at the tips of Blades 2 and 3 are the same. This is because the geometrical configurations and locations of Blades 2 and 3 are the same as shown in Fig. 2 and the same excitations are applied on these two blades, which in turn lead to the same structural responses. The result also shows that the maximum flapwise displacement on Blade 1 is smaller than that on Blades 2 and 3, this is because Blade 1 is lower than Blades 2 and 3 (see Fig. 2), and the wind loads on Blade 1 are smaller than those on Blades 2 and 3. Fig. 18 also shows that the maximum displacements are much larger when the blades are rotating and the corresponding values are 1.074, 1.338, $1.274 \mathrm{~m}$

540 respectively. When the wind turbine is in the operating condition, the flapwise vibrations of the blades are not the same and they are influenced by the original locations of the blades. 


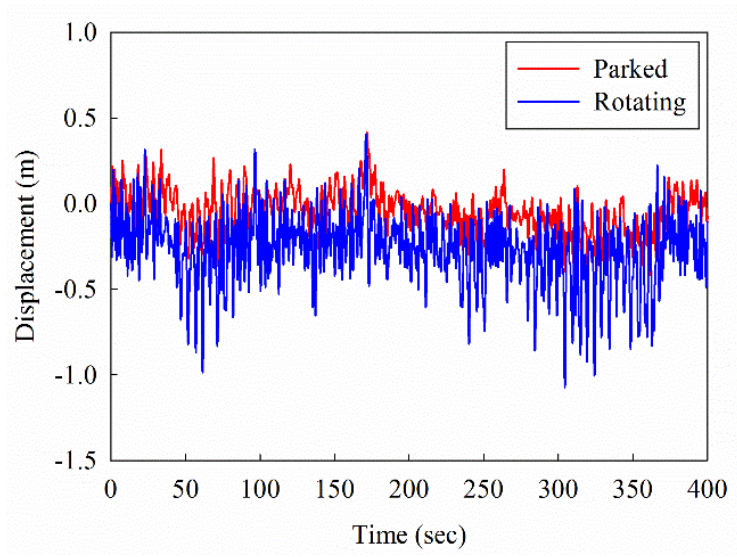

(a) Blade 1

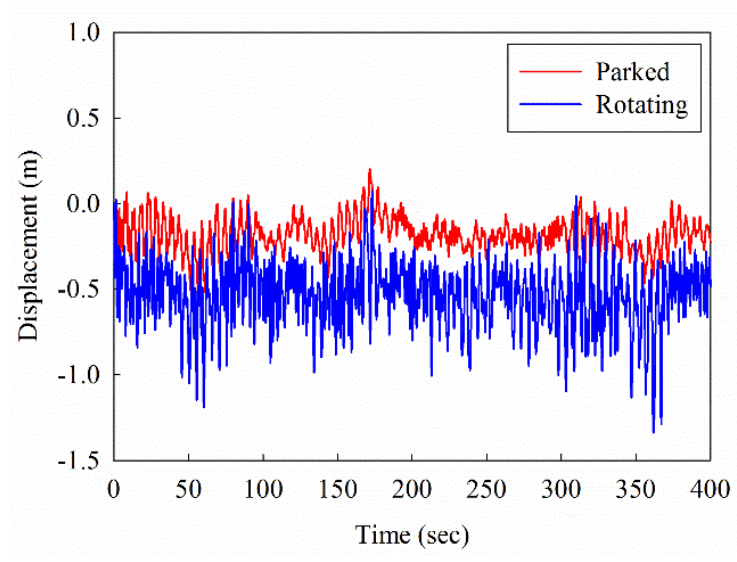

(b) Blade 2

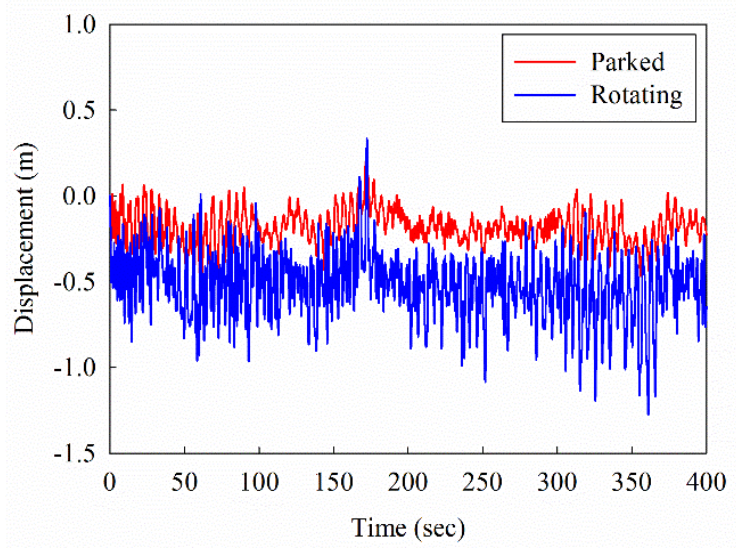

(c) Blade 3

545 Fig. 19 shows the edgewise displacements of the blades when they are in the parked and operating 546 conditions. Again, the non-zero baseline of the edgewise displacements is due to the contribution of 547 the mean term of the wind loads acting on the blades. As shown in Fig. 19(a), the maximum edgewise 548 displacements at the tips of Blades 1, 2 and 3 are 0.067, 0.138 and $0.138 \mathrm{~m}$ respectively when the 549 turbine is in the parked condition. When the blades are rotating, as shown in Fig. 19(b), the edgewise responses of the three blades are identical because of the same geometrical and structural parameters in the rotor plane. Compared to the parked condition, the edgewise displacements of the blades are 552 much larger when they are rotating. The edgewise displacements at the tips of the blades experience a 553 sinusoidal variation in magnitude after about $25 \mathrm{~s}$ and the frequency of this variation equals to the rotor frequency of $0.202 \mathrm{~Hz}(1.27 \mathrm{rad} / \mathrm{s})$. At the first $25 \mathrm{~s}$, the amplitudes of the edgewise 


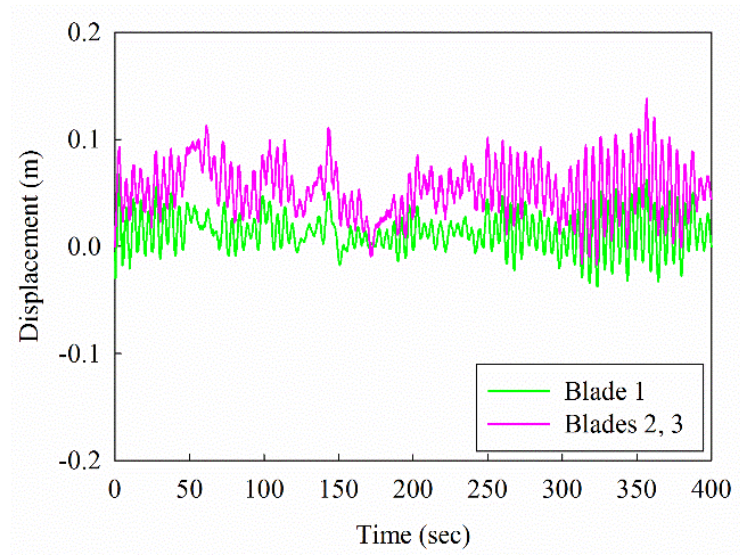

(a) Parked

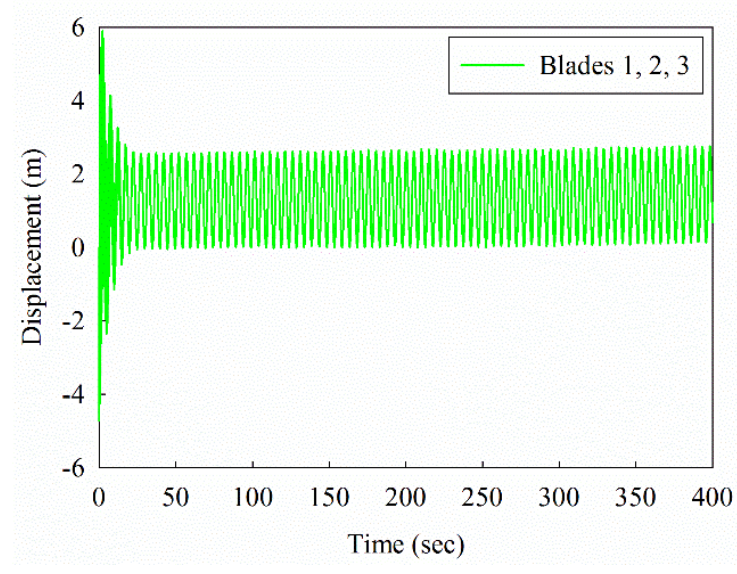

(b) Rotating

Fig. 19. Edgewise displacement time histories at the blade tips

To further explain the above results, Fig. 20 shows the PSDs of the acceleration responses at the tip of

Blade 2 in the flapwise and edgewise directions respectively. The acceleration PSDs of other blades are similar with those of Blade 2, they are therefore not shown for brevity. As shown in Fig. 20(a), three obvious peaks appear at $0.204,0.544$ and $0.603 \mathrm{~Hz}$ respectively when the wind turbine is in the parked condition. The first peak corresponds to the first fore-aft vibration mode of the tower, because of the interaction between the tower and blades as discussed above. Another two peaks correspond to the first flapwise pitch and collective flap vibration modes of the blades as shown in Table 5 in Section 4.2. It should be noted that the first flapwise yaw vibration mode of the blades is not excited since the locations of the blades and wind loads acting on the blades are symmetric and the flapwise yaw vibration mode is antisymmetric as shown in Fig. 6. The antisymmetric vibration mode cannot be excited by the symmetric load when it is acting on a symmetric structure. When the blades are rotating, the three peaks occur at $0.204,0.565$ and $0.647 \mathrm{~Hz}$ respectively. Again the last two vibration frequencies cannot be obtained from Table 5 due to the blades rotation cannot be directly considered 
575

576

577

578

579

580

581

582

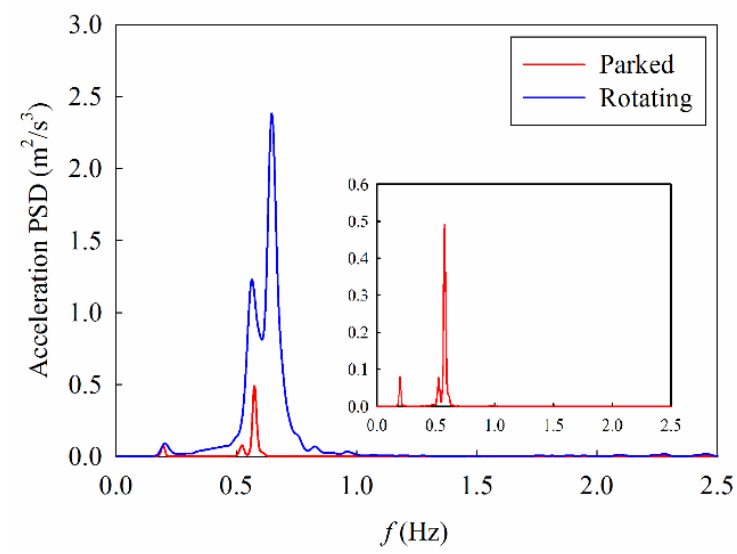

(a) Flapwise

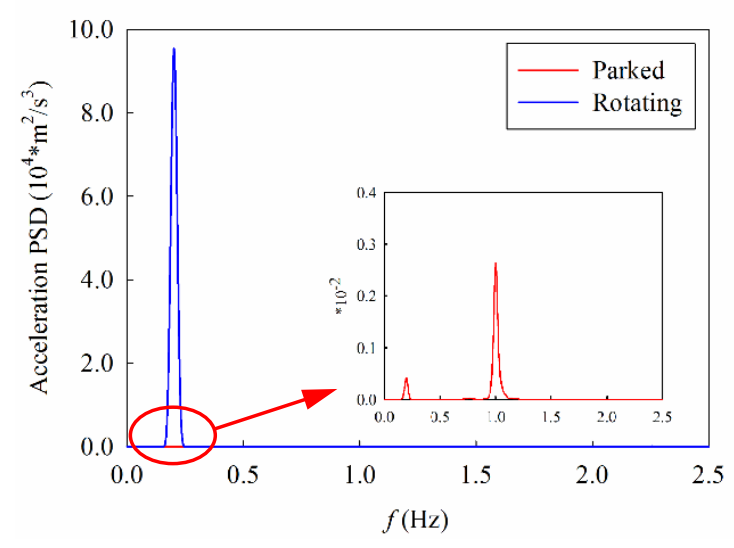

(b) Edgewise

Fig. 20. Flapwise and edgewise acceleration PSDs at the tip of Blade 2

\subsection{Influence of SSI}

To investigate the effect of SSI on the dynamic behaviours of the wind turbine, three different soils with undrained shear strength of $s_{\mathrm{u}}=25,50$ and $100 \mathrm{kPa}$ are considered in the present study and are used to represent the typical soft, medium and stiff soils. As discussed above, the rotation of the blades cannot be explicitly considered in the modal analysis by using ABAQUS, only the parked condition is considered when the vibration characteristics (vibration frequencies and vibration modes) of the wind turbine are calculated. Table 5 tabulates the vibration frequencies of the wind turbine without and with the consideration of SSI. The corresponding differences between the vibration 
597 is because the monopile inserts into the sea bed and is surrounded by the soil when SSI is considered,

598 which makes the tower more flexible compared to the case of the wind turbine fully fixed at the sea

599 bed level. Since the stiffness of the tower becomes smaller, the vibration frequencies of the tower

600 therefore decrease. For example, when the undrained shear strength of the soil $s_{\mathrm{u}}$ is $25 \mathrm{kPa}$, the first

601 vibration frequency of the tower in the fore-aft direction is $0.141 \mathrm{~Hz}$, and it is $0.204 \mathrm{~Hz}$ when SSI is

602 not considered. The reduction ratio reaches 30.9\%. It should be noted that the energy of the wind load

603 concentrates within the range of $0-0.1 \mathrm{~Hz}$ as shown in Fig. 7, when SSI is considered, the first

604 vibration frequency of the tower is closer to the dominant frequency of the wind load. In this case,

605 resonance might occur and larger structural responses are expected. Therefore, SSI should be considered to more accurately predict the dynamic responses of the wind turbine.

607

\section{$608 \quad$ Table 5}

609 Vibration frequencies of the wind turbine without and with SSI

\begin{tabular}{|c|c|c|c|c|c|c|c|c|}
\hline \multirow{2}{*}{ Mode } & \multirow{2}{*}{ Description } & \multirow{2}{*}{$\begin{array}{l}\mathrm{w} / \mathrm{o} \\
\mathrm{SSI} \\
(\mathrm{Hz})\end{array}$} & \multicolumn{2}{|c|}{$S_{\mathrm{u}}=25 \mathrm{kPa}$} & \multicolumn{2}{|c|}{$S_{\mathrm{u}}=50 \mathrm{kPa}$} & \multicolumn{2}{|c|}{$s_{\mathrm{u}}=100 \mathrm{kPa}$} \\
\hline & & & $\begin{array}{c}\text { Frequency } \\
(\mathrm{Hz})\end{array}$ & $\begin{array}{l}\text { Difference } \\
(\%)\end{array}$ & $\begin{array}{l}\text { Frequency } \\
(\mathrm{Hz})\end{array}$ & $\begin{array}{l}\text { Difference } \\
(\%)\end{array}$ & $\begin{array}{l}\text { Frequency } \\
(\mathrm{Hz})\end{array}$ & $\begin{array}{c}\text { Difference } \\
(\%)\end{array}$ \\
\hline 1 & $\begin{array}{l}\text { tower fore-aft } \\
\left.\text { (1 } 1^{\text {st }} \text { order }\right)\end{array}$ & 0.204 & 0.141 & -30.9 & 0.154 & -24.5 & 0.163 & -20.1 \\
\hline 2 & $\begin{array}{l}\text { tower side-to- } \\
\left.\text { side ( } 1^{\text {st }} \text { order }\right)\end{array}$ & 0.208 & 0.142 & -31.7 & 0.156 & -25.0 & 0.165 & -20.7 \\
\hline 3 & $\begin{array}{l}\text { blade flapwise } \\
\left.\text { yaw ( } 1^{\text {st }} \text { order }\right)\end{array}$ & 0.488 & 0.474 & -2.9 & 0.474 & -2.9 & 0.474 & -2.9 \\
\hline 4 & $\begin{array}{l}\text { blade flapwise } \\
\text { pitch ( }\left(1^{\text {st }} \text { order) }\right.\end{array}$ & 0.544 & 0.532 & -2.2 & 0.536 & -1.5 & 0.538 & -1.1 \\
\hline 5 & $\begin{array}{l}\text { blade collective } \\
\left.\text { flap ( } 1^{\text {st }} \text { order }\right)\end{array}$ & 0.603 & 0.594 & -1.5 & 0.596 & -1.2 & 0.598 & -0.8 \\
\hline 6 & $\begin{array}{l}\text { blade edgewise } \\
\text { pitch ( }\left(1^{\text {st }} \text { order) }\right.\end{array}$ & 1.176 & 1.189 & 1.1 & 1.206 & 2.6 & 1.145 & -2.6 \\
\hline 7 & $\begin{array}{l}\text { blade edgewise } \\
\left.\text { yaw ( } 1^{\text {st }} \text { order }\right)\end{array}$ & 1.206 & 1.231 & 2.1 & 1.250 & 3.6 & 1.154 & -4.3 \\
\hline 8 & $\begin{array}{l}\text { tower fore-aft } \\
\left.\text { ( } 2^{\text {nd }} \text { order }\right)\end{array}$ & 1.562 & 1.036 & -33.7 & 1.121 & -28.2 & 1.242 & -20.5 \\
\hline 9 & $\begin{array}{l}\text { tower side-to- } \\
\left.\text { side ( } 2^{\text {nd }} \text { order }\right)\end{array}$ & 1.630 & 1.003 & -38.5 & 1.096 & -32.8 & 1.279 & -21.5 \\
\hline 10 & $\begin{array}{l}\text { blade flapwise } \\
\text { yaw ( }\left(2^{\text {nd }} \text { order }\right)\end{array}$ & 1.700 & 1.625 & -4.4 & 1.626 & -4.4 & 1.626 & -4.4 \\
\hline
\end{tabular}


611 Different from modal analysis, the wind turbine is assumed in the operating condition when the 612 structural responses are calculated. In this section, a rotor angular velocity of $\Omega=1.27 \mathrm{rad} / \mathrm{s}$ is 613 considered.

614 Fig. 21 shows the displacement time histories at the top of the tower in the fore-aft and side-to-side 615 directions without and with the consideration of SSI. Table 6 tabulates the maximum fore-aft and 616 side-to-side displacements at the tower top, and the corresponding differences between the peak 617 displacements without and with SSI are given in the table as well. As shown in Fig. 21, the tower 618 vibrations are much larger in both the fore-aft and side-to-side directions when SSI is considered, and 619 with the increment of soil shear strength, the lateral stiffness of soil increases and the deflection of monopile decreases [36]. As shown in Fig. 21(a) and Table 6, the maximum fore-aft displacements at the top of the tower are 1.742, 1.383 and $1.297 \mathrm{~m}$ respectively when $s_{\mathrm{u}}=25,50$ and $100 \mathrm{kPa}$, which increase by $158.5 \%, 105.2 \%$ and $92.4 \%$ respectively compared to that when the wind turbine is fixed at the sea bed level, i.e. neglecting the interaction between the monopile foundation and the surrounding soil. Fig. 21(b) and Table 6 also show that the peak displacements at the tower top in the side-to-side direction are $0.388,0.301$ and $0.269 \mathrm{~m}$ respectively. Compared to the case in which SSI is not considered, these values are increased by $88.3 \%, 46.1 \%$ and $30.6 \%$.

627

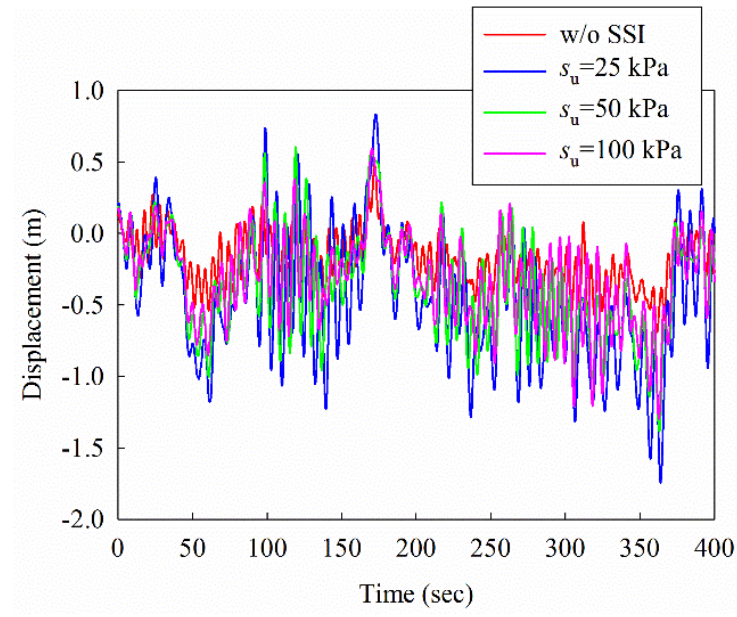

(a) Fore-aft

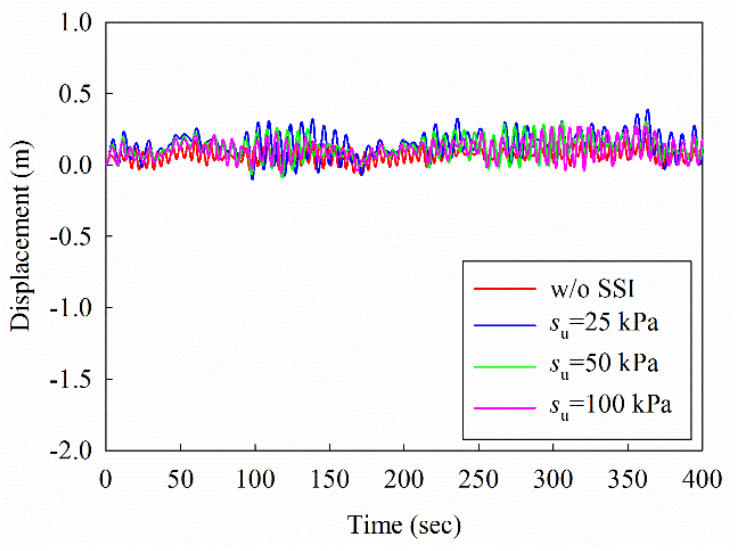

(b) Side-to-side 
Maximum displacements at the tower top without and with SSI

\begin{tabular}{|c|c|c|c|c|c|c|c|}
\hline \multirow[b]{2}{*}{ Direction } & \multirow{2}{*}{$\begin{array}{l}\text { w/o } \\
\text { SSI } \\
\text { (m) }\end{array}$} & \multicolumn{2}{|c|}{$S_{\mathrm{u}}=25 \mathrm{kPa}$} & \multicolumn{2}{|c|}{$s_{\mathrm{u}}=50 \mathrm{kPa}$} & \multicolumn{2}{|c|}{$S_{\mathrm{u}}=100 \mathrm{kPa}$} \\
\hline & & $\begin{array}{c}\text { Displacement } \\
(\mathrm{m})\end{array}$ & $\begin{array}{c}\text { Difference } \\
(\%)\end{array}$ & $\begin{array}{c}\text { Displacement } \\
\text { (m) }\end{array}$ & $\begin{array}{c}\text { Difference } \\
(\%)\end{array}$ & $\begin{array}{c}\text { Displacement } \\
\text { (m) }\end{array}$ & $\begin{array}{c}\text { Difference } \\
(\%)\end{array}$ \\
\hline Fore-aft & 0.674 & 1.742 & 158.5 & 1.383 & 105.2 & 1.297 & 92.4 \\
\hline $\begin{array}{l}\text { Side-to- } \\
\text { side }\end{array}$ & 0.206 & 0.388 & 88.3 & 0.301 & 46.1 & 0.269 & 30.6 \\
\hline
\end{tabular}

632

Fig. 22 shows the PSDs of the acceleration responses at the top of the tower in the fore-aft and sidethey are $0.141,0.154$ and $0.163 \mathrm{~Hz}$ respectively when $s_{\mathrm{u}}=25,50$ and $100 \mathrm{kPa}$. It should be noted that the second peak appearing at $0.647 \mathrm{~Hz}$ corresponds to the first collective flap vibration mode of the the observations in Table 5 that SSI has almost no effect on the vibration frequencies of the blades. Similarly, as shown in Fig. 22(b), the first vibration frequency of the tower in the side-to-side

641 direction decreases as well when the effect of SSI is included. The corresponding vibration 642 frequencies are $0.142,0.156$ and $0.165 \mathrm{~Hz}$ respectively when $s_{\mathrm{u}}=25,50$ and $100 \mathrm{kPa}$. Fig. 22 also shows that more energies are obtained for the softer soil, which in turn result in the larger tower responses as shown in Fig. 21.

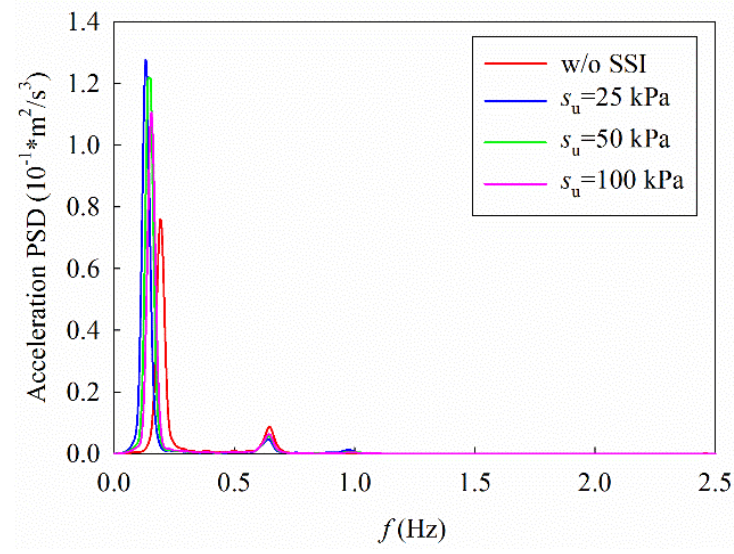

(a) Fore-aft

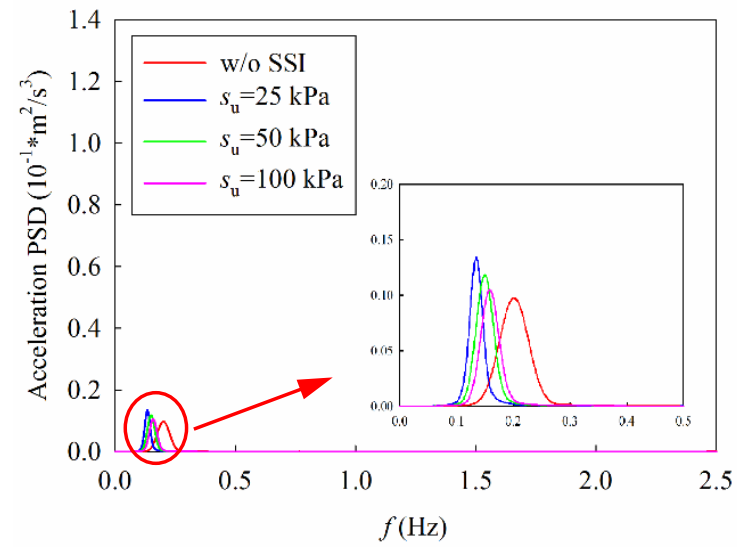

(b) Side-to-side 
647 Fig. 23 shows the displacement time histories at the tips of the blades in the flapwise direction. Table

6487 tabulates the maximum flapwise displacements at each blade tip and the corresponding differences 649 when SSI is considered or not. As shown in Table 7, the largest displacements at the tip of Blade 1 are 1.536, 1.231 and $1.220 \mathrm{~m}$ respectively when $s_{\mathrm{u}}=25,50$ and $100 \mathrm{kPa}$. For Blade 2, the maximum values are 1.745, 1.648 and $1.632 \mathrm{~m}$ respectively, and the corresponding values are $1.701,1.529$ and $1.486 \mathrm{~m}$ respectively for Blade 3. It can be seen that the flapwise displacement responses of the blades are influenced by SSI but the extents are smaller than those of the tower (refer to Table 6). This is because the soil springs are directly connected to the monopile foundation of the tower while the influence of SSI on the blades is mainly through the (indirect) interaction between the tower and blades. As discussed above, softer soil leads to larger fore-aft vibrations of the tower, which in turn leads to the more severe interaction between the tower and blades, and therefore the larger flapwise displacements of the blades.

Fig. 24 shows the edgewise displacement time histories at the tip of Blade 2, and the displacements at other two blades are not shown since they are the same as discussed above. As shown, again the edgewise displacements of the rotating blades are governed by the rotor rotation and SSI has a negligible effect on the displacement responses of the blades in the edgewise direction. This is because the vibrations of the tower in the side-to-side direction are very small as shown in Fig. 21(b), which results in the negligible interactions between the tower and blades in the side-to-side direction. The results are consistent with those reported by Fitzgerald and Basu [17]. Fig. 25 shows the PSDs of the acceleration responses at the tip of Blade 2 in the flapwise and edgewise directions. As shown in Fig. 25(a), three obvious peaks appear in the PSD curves and they correspond to the first fore-aft vibration mode of the tower, the first flapwise pitch and collective flap vibration modes of the blades respectively as discussed in Section 4.1. As shown, the frequency corresponding to the first peak changes when SSI is considered and the value becomes smaller when softer soil is considered. This is easy to understand, when softer soil is considered, the system becomes more flexible. For the second and third peaks, they correspond to the vibration modes of the blades and they are almost not influenced by SSI. Fig. 25(b) shows that only one peak occurs at the rotor frequency of the blades $(0.202 \mathrm{~Hz})$ and the energies included in the PSD curves are the same 
677

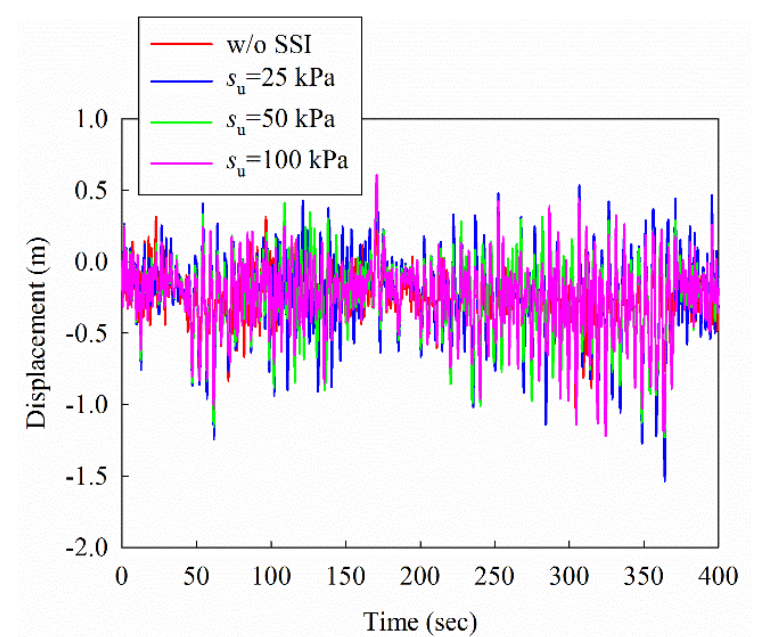

(a) Blade 1

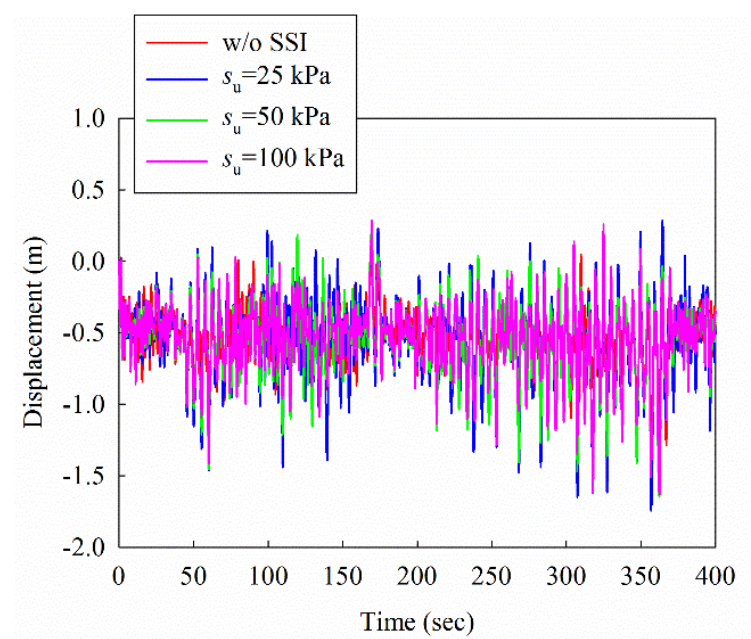

(b) Blade 2

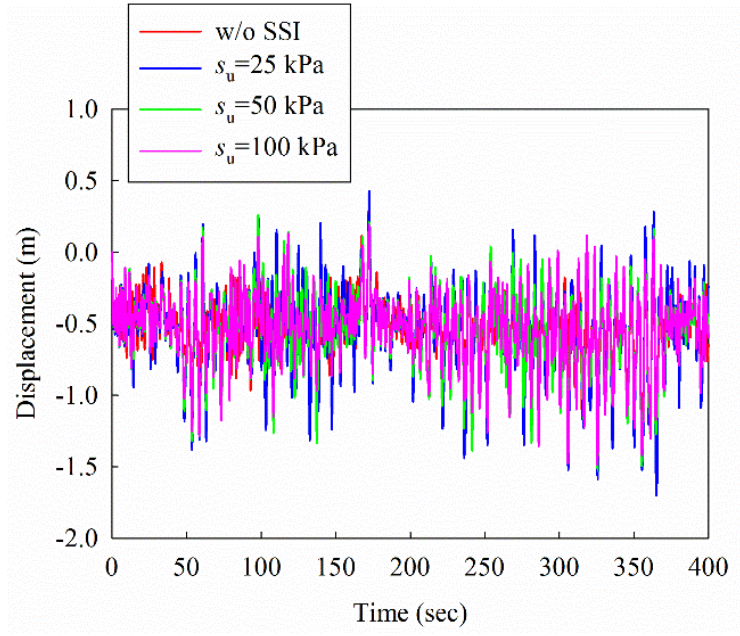

(c) Blade 3

Fig. 23. Flapwise displacement time histories at the blade tips without and with SSI

679

Table 7

Maximum flapwise displacements at the blade tip without and with SSI

\begin{tabular}{cccccccc}
\hline Location & $\begin{array}{c}\text { W/o } \\
\text { SSI } \\
(\mathrm{m})\end{array}$ & $\begin{array}{c}\text { Displacement } \\
(\mathrm{m})\end{array}$ & $\begin{array}{c}\text { Difference } \\
(\%)\end{array}$ & $\begin{array}{c}\text { Displacement } \\
(\mathrm{m})\end{array}$ & $\begin{array}{c}\text { Difference } \\
(\%)\end{array}$ & $\begin{array}{c}S_{\mathrm{u}}=50 \mathrm{kPa} \\
\text { Displacement } \\
(\mathrm{m})\end{array}$ & $\begin{array}{c}S_{\mathrm{u}}=100 \mathrm{kPa} \\
\text { Difference } \\
(\%)\end{array}$ \\
\hline $\begin{array}{c}\text { Tip of } \\
\text { Blade 1 }\end{array}$ & 1.074 & 1.536 & 43.0 & 1.231 & 14.6 & 1.220 & 13.6 \\
$\begin{array}{c}\text { Tip of } \\
\text { Blade 2 }\end{array}$ & 1.338 & 1.745 & 30.4 & 1.648 & 23.2 & 1.632 & 22.0 \\
$\begin{array}{c}\text { Tip of } \\
\text { Blade 3 }\end{array}$ & 1.274 & 1.701 & 33.5 & 1.529 & 20.0 & 1.486 & 16.6 \\
\hline
\end{tabular}


683

684

685

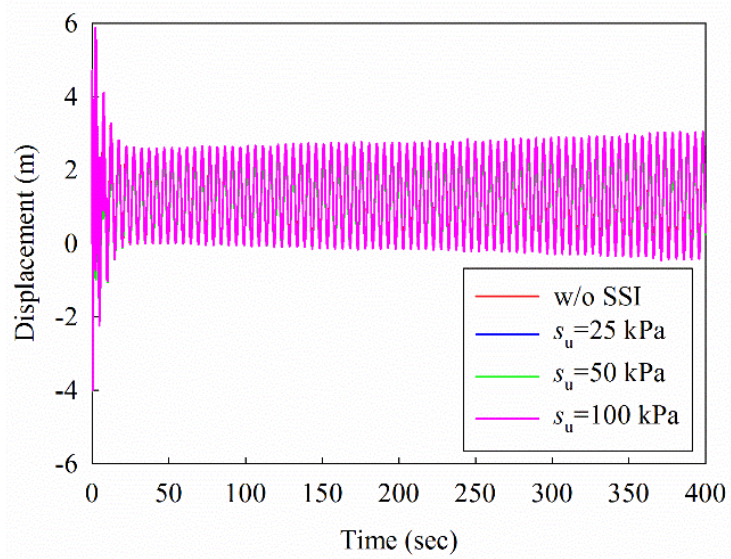

Fig. 24. Edgewise displacement time histories at the tip of Blade 2 without and with SSI

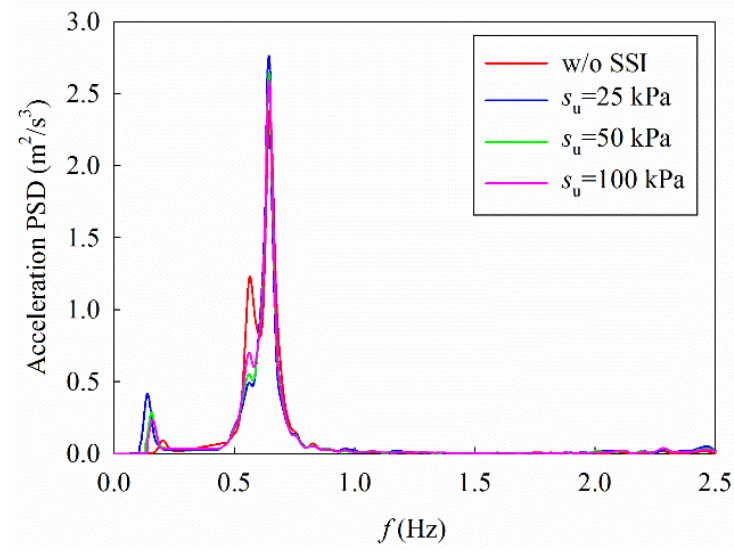

(a) Flapwise

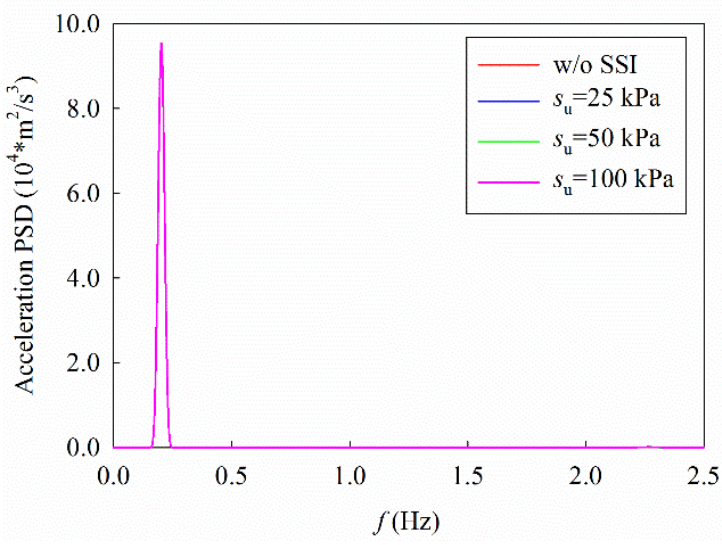

(b) Edgewise

Fig. 25. Flapwise and edgewise acceleration PSDs at the tip of Blade 2 without and with SSI

\subsection{Influence of rotor velocity}

689

The rotor velocity may also significantly influence the structural responses. The designed cut-in and rated rotor velocities of the NREL 5 MW wind turbine are 6.9 and 12.1 rpm respectively as tabulated in Table 1. In other words, the wind turbine starts to rotate at a (cut-in) rotor speed of $6.9 \mathrm{rpm}$ and the maximum energy output of the wind turbine will be achieved at a (rated) rotor speed of $12.1 \mathrm{rpm}$. To examine the influence of rotor velocity, the rotor velocity of $8 \mathrm{rpm}(0.84 \mathrm{rad} / \mathrm{s})$ and $12.1 \mathrm{rpm}(1.27$ $\mathrm{rad} / \mathrm{s}$ ) are investigated, which are within the designed rotor velocity range. Another rotor velocity of $16 \mathrm{rpm}(1.68 \mathrm{rad} / \mathrm{s})$ is also considered to represent a worst rotating condition in the present study. SSI is also considered in this section and the undrained shear strength of soil is taken as $50 \mathrm{kPa}$. 
As discussed in Section 3.2, the wind loads on the blades are influenced by the rotor velocity. Fig. 26 shows the simulated wind loads on Blade 2 in the flapwsie and edgewise directions when the rotor velocities are 0.84 and $1.68 \mathrm{rad} / \mathrm{s}$ respectively. Together with the wind loads shown in Fig. 13(b) (the wind loads when $\Omega=1.27 \mathrm{rad} / \mathrm{s}$ ), it can be seen that larger rotor velocity results in larger wind loads acting on the blade. For conciseness, the wind loads acting on the other two blades are not plotted in the figure. Similar trend is obtained.

Fig. 27 shows the displacement time histories at the top of the tower in the fore-aft and side-to-side directions and the maximum displacements are tabulated in Table 8. As shown in Fig. 27 and Table 8, the displacement responses at the top of the tower are increased in both directions with the increment of rotor velocity. The maximum fore-aft displacements at the tower top are 1.226, 1.383 and $1.523 \mathrm{~m}$ respectively when $\Omega=0.84,1.27$ and $1.68 \mathrm{rad} / \mathrm{s}$, and the corresponding values in the side-to-side direction are $0.258,0.301$ and $0.329 \mathrm{~m}$. This is because wind loads acting on the blades are larger with the increment of the rotor velocity as discussed above, and larger loads result in larger structural responses.

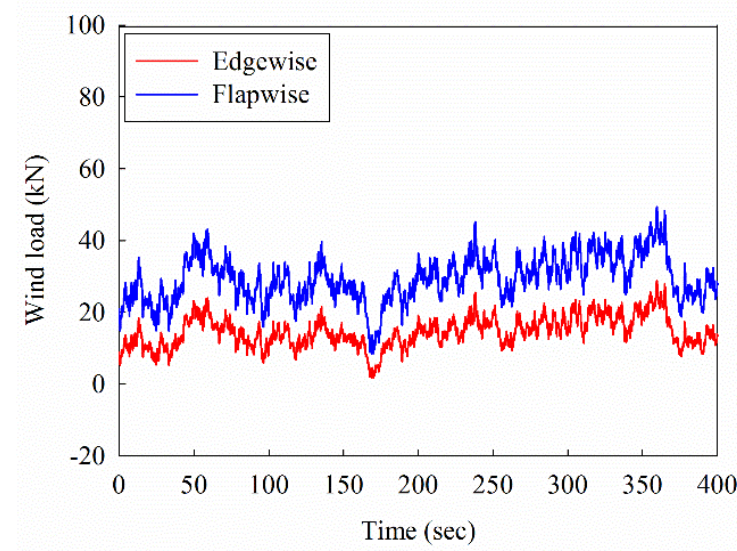

(a) $\Omega=0.84 \mathrm{rad} / \mathrm{s}$

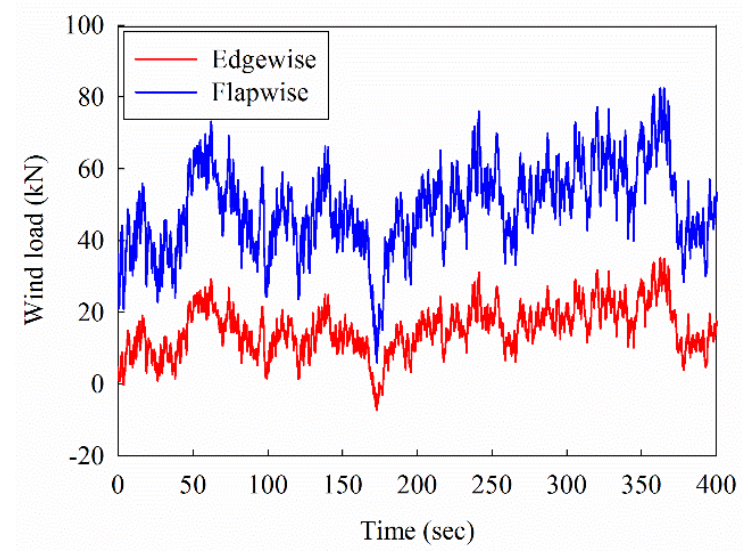

(b) $\Omega=1.68 \mathrm{rad} / \mathrm{s}$ 


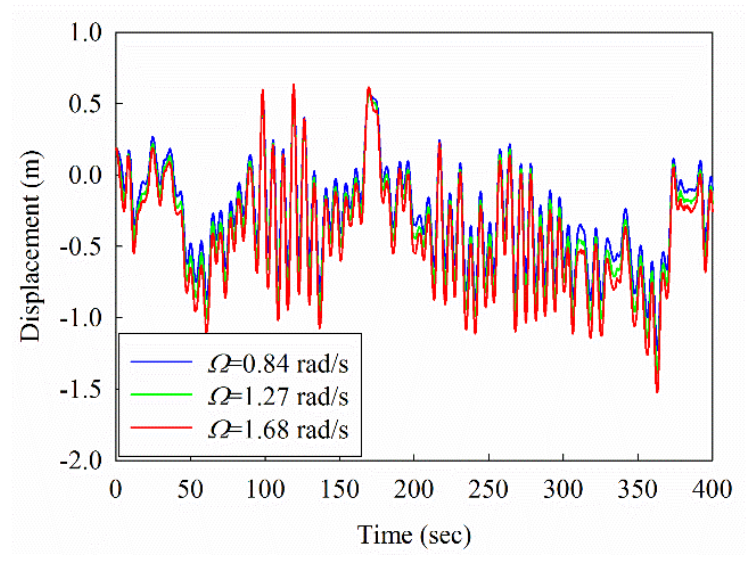

(a) Fore-aft

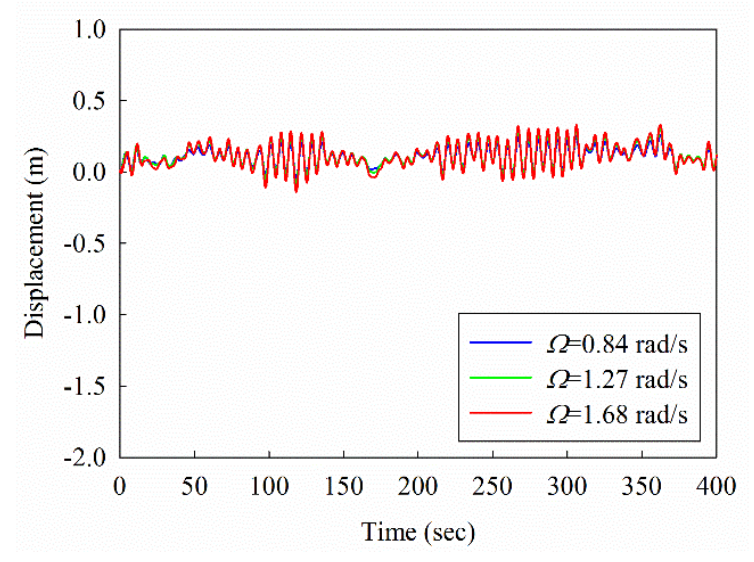

(b) Side-to-side

Fig. 27. Fore-aft and side-to-side displacement time histories at the tower top under different rotor velocities

\section{Table 8}

Maximum displacements at the tower top under different rotor velocities (Unit: m)

\begin{tabular}{cccc}
\hline Direction & $\Omega=0.84 \mathrm{rad} / \mathrm{s}$ & $\Omega=1.27 \mathrm{rad} / \mathrm{s}$ & $\Omega=1.68 \mathrm{rad} / \mathrm{s}$ \\
\hline Fore-aft & 1.226 & 1.383 & 1.523 \\
Side-to-side & 0.258 & 0.301 & 0.329 \\
\hline
\end{tabular}

717

718 Fig. 28 shows the PSDs of the fore-aft and side-to-side acceleration responses at the top of the tower.

719 As shown in Fig. 28(a), the first peak appears at $0.154 \mathrm{~Hz}$, which corresponds to the first fore-aft vibration frequency of the tower and is not influenced by the rotor velocity. The second peak corresponds to the first collective flap vibration mode of the blades and the frequencies are 0.598, 0.647 and $0.684 \mathrm{~Hz}$ respectively when $\Omega=0.84,1.27$ and $1.68 \mathrm{rad} / \mathrm{s}$, which are $0.4 \%, 8.1 \%$ and $14.7 \%$ greater than the frequency of $0.596 \mathrm{~Hz}$ when the wind turbine is in the parked condition as tabulated in Table 5. This is because the geometric stiffness arising out of centrifugal stiffening in the flapwise direction can increase the stiffness and vibration frequencies of the blades [10, 33]. In the side-to-side direction, only one peak occurs at $0.156 \mathrm{~Hz}$ as shown in Fig. 28(b). This value corresponds to the first vibration frequency of the tower in the side-to-side direction as tabulated in Table 5. Fig. 28 also shows that more energies concentrate in the PSD curves when the blades rotate at a larger velocity, which in turn lead to larger tower responses as shown in Fig. 27. 


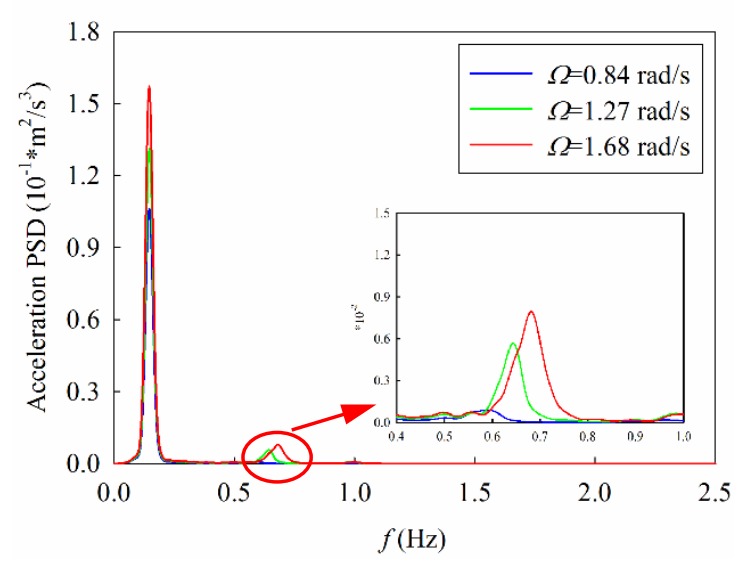

(a) Fore-aft

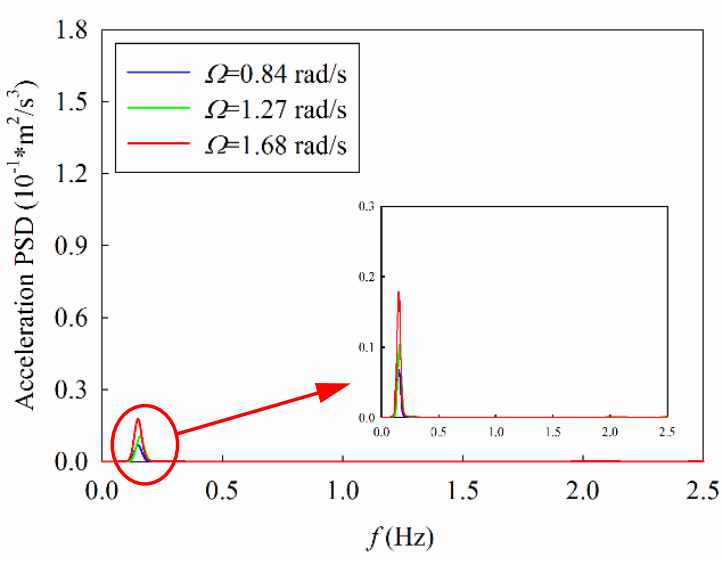

(b) Side-to-side

Fig. 28. Fore-aft and side-to-side acceleration PSDs at the tower top under different rotor velocities

732

733

Fig. 29 shows the displacement time histories at the tips of blades in the flapwise direction and Fig. 30 shows the edgewise displacements at the tip of Blade 2. As shown in Figs. 29 and 30, both the flapwise and edgewise displacement responses of the blades increase with the increment of the rotor velocity. This is because, as discussed above, the wind loads acting on the blades are larger as shown in Figs. 13(b) and 26, which in turn lead to the larger responses of the blades. Table 9 tabulates the maximum flapwise displacements at each blade tip. The maximum displacements at the tip of Blade 1 in the flapwise direction are 1.186, 1.124 and 1.155 m respectively when $\Omega=0.84,1.27$ and $1.68 \mathrm{rad} / \mathrm{s}$; for Blade 2, the peak values are 1.231, 1.648 and 1.529 m respectively; and the corresponding values are 1.352, 1.773 and $1.984 \mathrm{~m}$ respectively for Blade 3. It is interesting to note that, as shown in Fig. 30, the displacements at the blade tip in the edgewise direction is not a constant as that shown in Fig. 19(b) but increase slightly from about $t=200 \mathrm{~s}$ especially when $\Omega=1.68 \mathrm{rad} / \mathrm{s}$. This is because the edgewise wind loads acting on the blades become larger in the time duration of 200-400 s as shown in Fig. 26.

Fig. 31 shows the PSDs of the acceleration responses at the tip of Blade 2 in the flapwise and edgewise directions. As shown in Fig. 31(a), the first peak corresponding to the first vibration frequency of the tower $(0.154 \mathrm{~Hz})$ is not influenced by the rotor velocity, while the second peak corresponds to the first flapwise pitch vibration mode of the blades and they are $0.549,0.565$ and $0.644 \mathrm{~Hz}$ respectively when $\Omega=0.84,1.27$ and $1.68 \mathrm{rad} / \mathrm{s}$; the third peak is related to the first collective 
751 flap vibration mode of the blades, which are 0.598, 0.647 and $0.684 \mathrm{~Hz}$ respectively. As shown in Fig.

752 31(b), only one obvious peak occurs and the frequencies are $0.134,0.202$ and $0.267 \mathrm{~Hz}$ respectively,

753 which are the rotor velocities (0.84, 1.27 and 1.68rad/s). Fig. 31 also clearly shows that much more

754 energies are included in the PSD curves with the increment of the rotor velocity, which lead to the

755 larger responses of the blades in both the flapwise and edgewise directions as shown in Figs. 29 and

75630

757

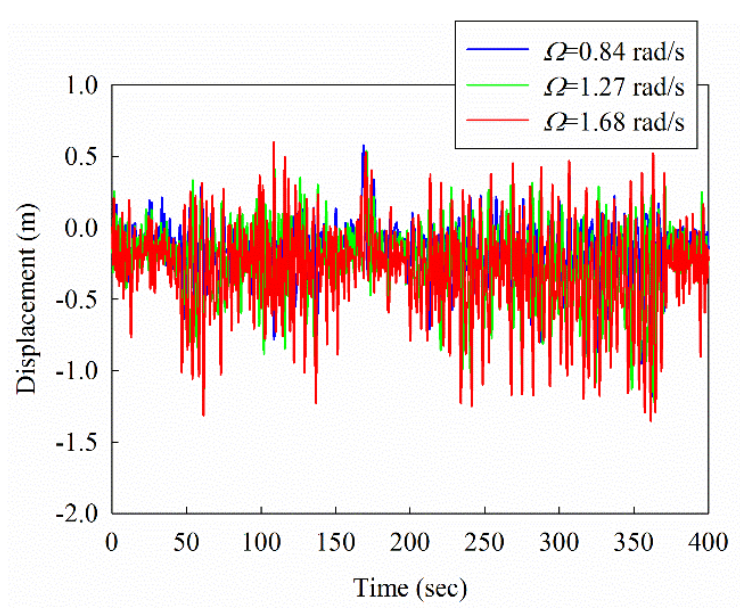

(a) Blade 1

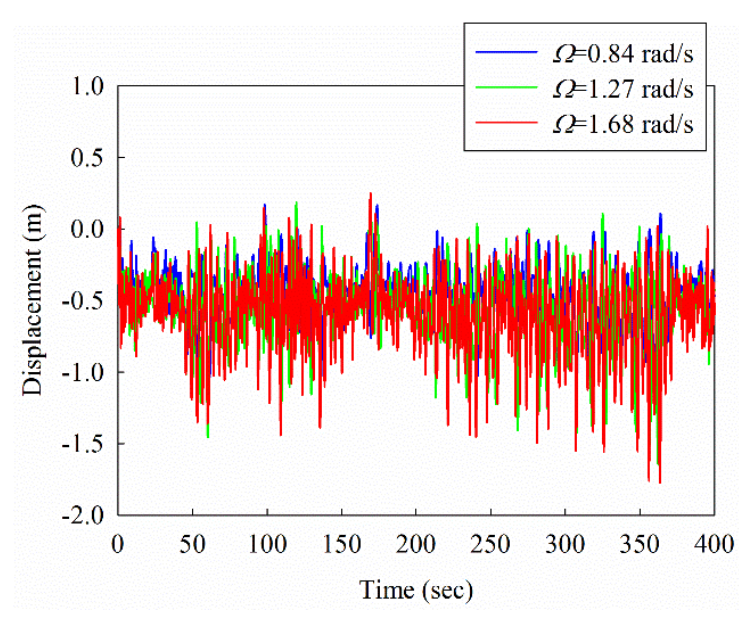

(b) Blade 2

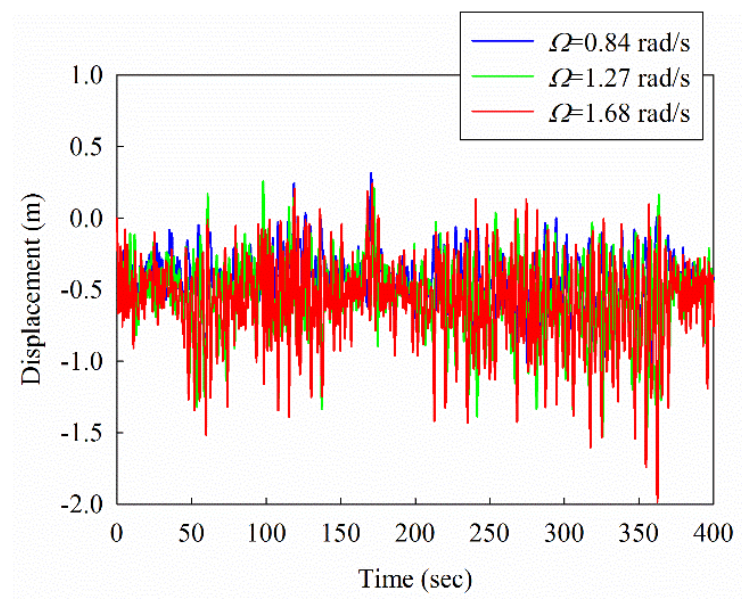

(c) Blade 3

Fig. 29. Flapwise displacement time histories at the blade tips under different rotor velocities 
763 Maximum flapwise displacements at the blade tip under different rotor velocities (Unit: m)

\begin{tabular}{cccc}
\hline Location & $\Omega=0.84 \mathrm{rad} / \mathrm{s}$ & $\Omega=1.27 \mathrm{rad} / \mathrm{s}$ & $\Omega=1.68 \mathrm{rad} / \mathrm{s}$ \\
\hline Tip of Blade 1 & 1.186 & 1.231 & 1.352 \\
Tip of Blade 2 & 1.124 & 1.648 & 1.773 \\
Tip of Blade 3 & 1.155 & 1.529 & 1.984 \\
\hline
\end{tabular}

764

765

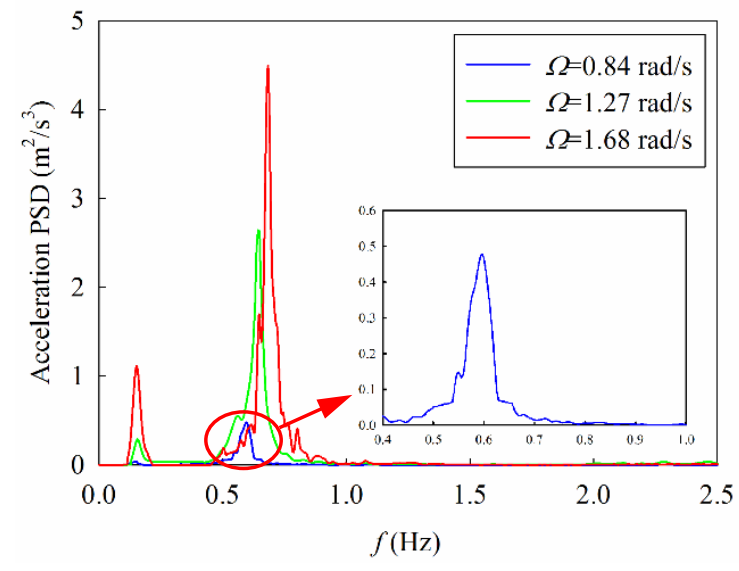

(a) Flapwise

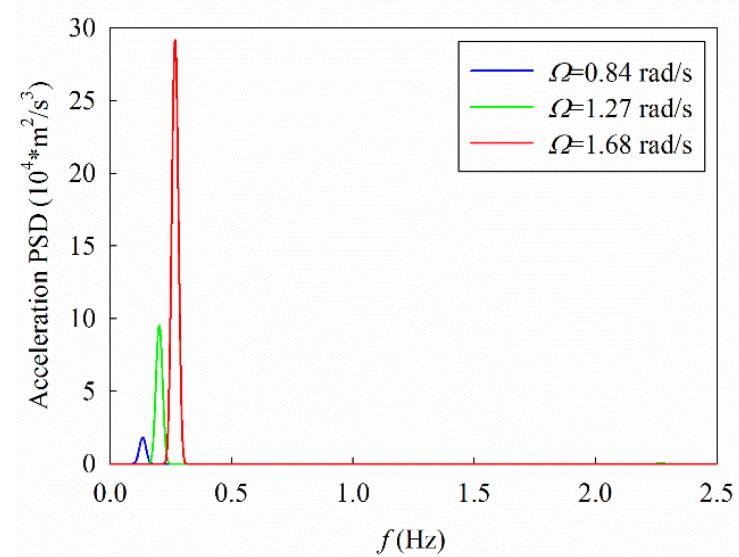

(b) Edgewise

Fig. 31. Flapwise and edgewise acceleration PSDs at the tip of Blade 2 under different rotor velocities

\section{Conclusions}

771 This paper carries out numerical studies on the dynamic responses of the NREL 5 MW wind turbine

772 subjected to the combined wind and sea wave loadings. The influences of operational conditions, soil- 
monopile interaction and rotor velocity on the tower and blades are systematically investigated.

774 Numerical results reveal that:

775 (1) The maximum displacements at the top of the tower in the fore-aft and side-to-side directions when the wind turbine rotates with a rotor velocity of $1.27 \mathrm{rad} / \mathrm{s}$ are $142 \%$ and $222 \%$ larger than those when the wind turbine is in the parked condition. The peak flapwise displacement of the blades is about 2.5 times of that of the parked wind turbine. Previous studies by assuming the wind turbines in the parked condition may result in non-conservative structural response estimations and unsafe design of structural components. In the current design codes, safety factors are normally used to account for the uncertainties and variabilities in loads, analysis methods and the importance of structural components for the wind turbines [48, 49]. It will be interesting to develop a uniform safety factor that can be used in the operating response estimation based on the parked results. However, more comprehensive research works are needed.

(2) The vibration frequencies of the tower are significantly decreased when SSI is considered, while SSI only marginally affects the natural frequencies of the blades. The out-of-rotor-plane displacement responses of the tower and blades are substantially influenced by SSI. However, SSI has a negligible effect on the displacements of the blades in the edgewise direction.

(3) The out-of-rotor-plane displacements of the tower and blades increase with the increment of the rotor velocity. The displacements of the blades in the edgewise direction increase slightly when the rotor velocity becomes larger.

792 It should be noted that wind is the dominant load in the present study by comparing Fig. 9 with Fig. 15. Moreover, sea wave is applied near to the bottom of the structure, the influence of the sea wave load on the structural responses is less evident compared to the wind load. When the wind turbine is located in the medium to deep water, the effect of the sea wave load might be obvious. Investigation of the influence of water depth on the structural responses is out of the scope of the present study. Moreover, all the above conclusions are obtained based on the latest NREL 5 MW wind turbine, which is the largest wind turbine in the world currently. These conclusions may only be applicable to this group of wind turbines. To apply the present results in engineering practice and to guide the 

turbines as well), more comprehensive analyses are needed.

\section{Acknowledgements}

The authors would like to acknowledge the support from Australian Research Council Discovery

Scholarship (CIPRS).

\section{References}

[1] Jonkman J, Butterfield S, Musial W, Scott G. Definition of a 5-MW reference wind turbine for offshore system development Technical Report No NREL/TP-500-38060. Golden, CO: National Renewable Energy Laboratory; 2009.

813 [2] Bazeos N, Hatzigeorgiou G, Hondros I, Karamaneas H, Karabalis D, Beskos D. Static, seismic and stability

[3] Lavassas I, Nikolaidis G, Zervas P, Efthimiou E, Doudoumis I, Baniotopoulos C. Analysis and design of the prototype of a steel 1-MW wind turbine tower. Eng Struct 2003;25:1097-106.

[4] Colwell S, Basu B. Tuned liquid column dampers in offshore wind turbines for structural control. Eng Struct 2009;31:358-68.

[5] Chen J, Georgakis CT. Tuned rolling-ball dampers for vibration control in wind turbines. J Sound Vib 2013;332:5271-82.

[6] Bisoi S, Haldar S. Dynamic analysis of offshore wind turbine in clay considering soil-monopile-tower interaction. Soil Dyn Earthquake Eng 2014;63:19-35.

[7] Bisoi S, Haldar S. Design of monopile supported offshore wind turbine in clay considering dynamic soilstructure-interaction. Soil Dyn Earthquake Eng 2015;73:103-17.

[8] Zuo H, Bi K, Hao H. Using multiple tuned mass dampers to control offshore wind turbine vibrations under multiple hazards. Eng Struct 2017;141:303-15.

[9] Hansen M. Aerodynamics of Wind Turbines. 2nd ed. London: Earthscan; 2008.

[10] Murtagh PJ, Basu B, Broderick BM. Along-wind response of a wind turbine tower with blade coupling subjected to rotationally sampled wind loading. Eng Struct 2005;27:1209-19.

[11] Prowell I, Veletzos M, Elgamal A, Restrepo J. Experimental and numerical seismic response of a $65 \mathrm{~kW}$ wind turbine. J Earthquake Eng 2009;13:1172-90.

[12] Kjørlaug RA, Kaynia AM. Vertical earthquake response of megawatt-sized wind turbine with soil-structure interaction effects. Earthquake Eng Struct Dyn 2015;44:2341-58.

[13] Santangelo F, Failla G, Santini A, Arena F. Time-domain uncoupled analyses for seismic assessment of land-based wind turbines. Eng Struct 2016;123:275-99.

[14] Prowell I, Elgamal A, Uang CM, Enrique Luco J, Romanowitz H, Duggan E. Shake table testing and numerical simulation of a utility-scale wind turbine including operational effects. Wind Energy 2014;17:9971016.

[15] Quilligan A, O’Connor A, Pakrashi V. Fragility analysis of steel and concrete wind turbine towers. Eng Struct 2012;36:270-82.

[16] Harte M, Basu B, Nielsen SRK. Dynamic analysis of wind turbines including soil-structure interaction. Eng Struct 2012;45:509-18.

[17] Fitzgerald B, Basu B. Structural control of wind turbines with soil structure interaction included. Eng Struct 2016;111:131-51.

[18] Yuan C, Chen J, Li J, Xu Q. Fragility analysis of large-scale wind turbines under the combination of seismic and aerodynamic loads. Renewable Energy. 2017;113:1122-34. 
[19] Kim DH, Lee SG, Lee IK. Seismic fragility analysis of 5 MW offshore wind turbine. Renewable Energy.

[20] Jonkman JM, Buhl Jr ML. FAST User's Guide. Golden, CO: National Renewable Energy Laboratory (NREL); 2005.

[21] Damgaard M, Zania V, Andersen LV, Ibsen LB. Effects of soil-structure interaction on real time dynamic response of offshore wind turbines on monopiles. Eng Struct 2014;75:388-401.

[22] Kuo YS, Achmus M, Abdel-Rahman K. Minimum embedded length of cyclic horizontally loaded monopiles. J Geotech Geoenviron Eng 2011;138:357-63.

[23] Mostafa YE, El Naggar MH. Response of fixed offshore platforms to wave and current loading including soil-structure interaction. Soil Dyn Earthquake Eng 2004;24:357-68.

[24] Andersen LV, Vahdatirad M, Sichani MT, Sørensen JD. Natural frequencies of wind turbines on monopile foundations in clayey soils-a probabilistic approach. Comput Geotech 2012;43:1-11.

[25] Arany L, Bhattacharya S, Macdonald JH, Hogan SJ. Closed form solution of eigen frequency of monopile supported offshore wind turbines in deeper waters incorporating stiffness of substructure and SSI. Soil Dyn Earthquake Eng 2016;83:18-32.

[26] Lombardi D, Bhattacharya S, Wood DM. Dynamic soil-structure interaction of monopile supported wind turbines in cohesive soil. Soil Dyn Earthquake Eng 2013;49:165-80.

[27] Bhattacharya S, Adhikari S. Experimental validation of soil-structure interaction of offshore wind turbines. Soil Dyn Earthquake Eng 2011;31:805-16.

[28] Haciefendioğlu K. Stochastic seismic response analysis of offshore wind turbine including fluid-structuresoil interaction. Struct Des Tall Spec Build 2012;21:867-78.

[29] Kooijman H, Lindenburg C, Winkelaar D, van der Hooft E. Aero-elastic modelling of the DOWEC 6 MW pre-design in PHATAS DOWEC-F1W2-HJK-01-046/9. 2003.

[30] Bi K, Hao H. Using pipe-in-pipe systems for subsea pipeline vibration control. Eng Struct 2016;109:75-84. [31] Burton T, Jenkins N, Sharpe D, Bossanyi E. Wind energy handbook. 2nd ed. John Wiley \& Sons; 2011.

[32] Det Norske Veritas (DNV). DNV-RP-C205: Environmental conditions and environmental loads. Norway: DNV; 2010.

[33] Fitzgerald B, Basu B. Cable connected active tuned mass dampers for control of in-plane vibrations of wind turbine blades. J Sound Vib 2014;333:5980-6004.

[34] American Petroleum Institute (API). Petroleum and natural gas industries-specific requirements for offshore structures. Part 4-geotechnical andfoundation design considerations ISO 19901-4:2003 (Modified). 2011.

[35] Det Norske Veritas (DNV). DNV-OS-J101: Design of offshore wind turbine structures. Copenhagen, Denmark: DNV; 2014.

[36] Ashour M, Norris G, Pilling P. Lateral loading of a pile in layered soil using the strain wedge model. J Geotech Geoenviron Eng 1998;124:303-15.

[37] Hu WH, Thöns S, Rohrmann RG, Said S, Rücker W. Vibration-based structural health monitoring of a wind turbine system. Part I: Resonance phenomenon. Eng Struct 2015;89:260-72.

[38] Valamanesh V, Myers A. Aerodynamic damping and seismic response of horizontal axis wind turbine towers. J Struct Eng 2014;140:04014090.

[39] Andersen L. Assessment of lumped-parameter models for rigid footings. Comput Struct 2010;88:1333-47.

[40] Chopra AK. Dynamics of Structures. 4th ed. New Jersey: Prentice Hall; 2012.

[41] Benowitz BA, Deodatis G. Simulation of wind velocities on long span structures: a novel stochastic wave based model. J Wind Eng Ind Aerodyn 2015;147:154-63.

[42] Huang G, Liao H, Li M. New formulation of Cholesky decomposition and applications in stochastic simulation. Probab Eng Mech 2013;34:40-7.

[43] Hao H, Oliveira C, Penzien J. Multiple-station ground motion processing and simulation based on SMART-1 array data. Nucl Eng Des 1989;111:293-310.

[44] $\mathrm{Bi} \mathrm{K}$, Hao H. Modelling and simulation of spatially varying earthquake ground motions at sites with varying conditions. Probab Eng Mech 2012;29:92-104.

[45] Hasselmann K, Barnett T, Bouws E, Carlson H, Cartwright D, Enke K, et al. Measurements of wind-wave growth and swell decay during the Joint North Sea Wave Project (JONSWAP). Hamburg: Deutches Hydrographisches Institut; 1973.

[46] Sorensen RM. Basic coastal engineering. New York: Springer Science \& Business Media; 2005.

[47] Staino A, Basu B. Dynamics and control of vibrations in wind turbines with variable rotor speed. Eng Struct 2013;56:58-67.

[48] IEC 61400-1. Wind turbines-Part 1: Design requirements. 3rd ed. Geneva, Switzerland: International Electrotechnical Commission; 2005.

[49] IEC 61400-3. Wind turbines-Part 3: Design requirements for offshore wind turbines. 1st ed. Geneva, Switzerland: International Electrotechnical Commission; 2009. 